Abbreviated title: Learned distractor suppression

\title{
Neural mechanisms underlying expectation-dependent inhibition of distracting information
}

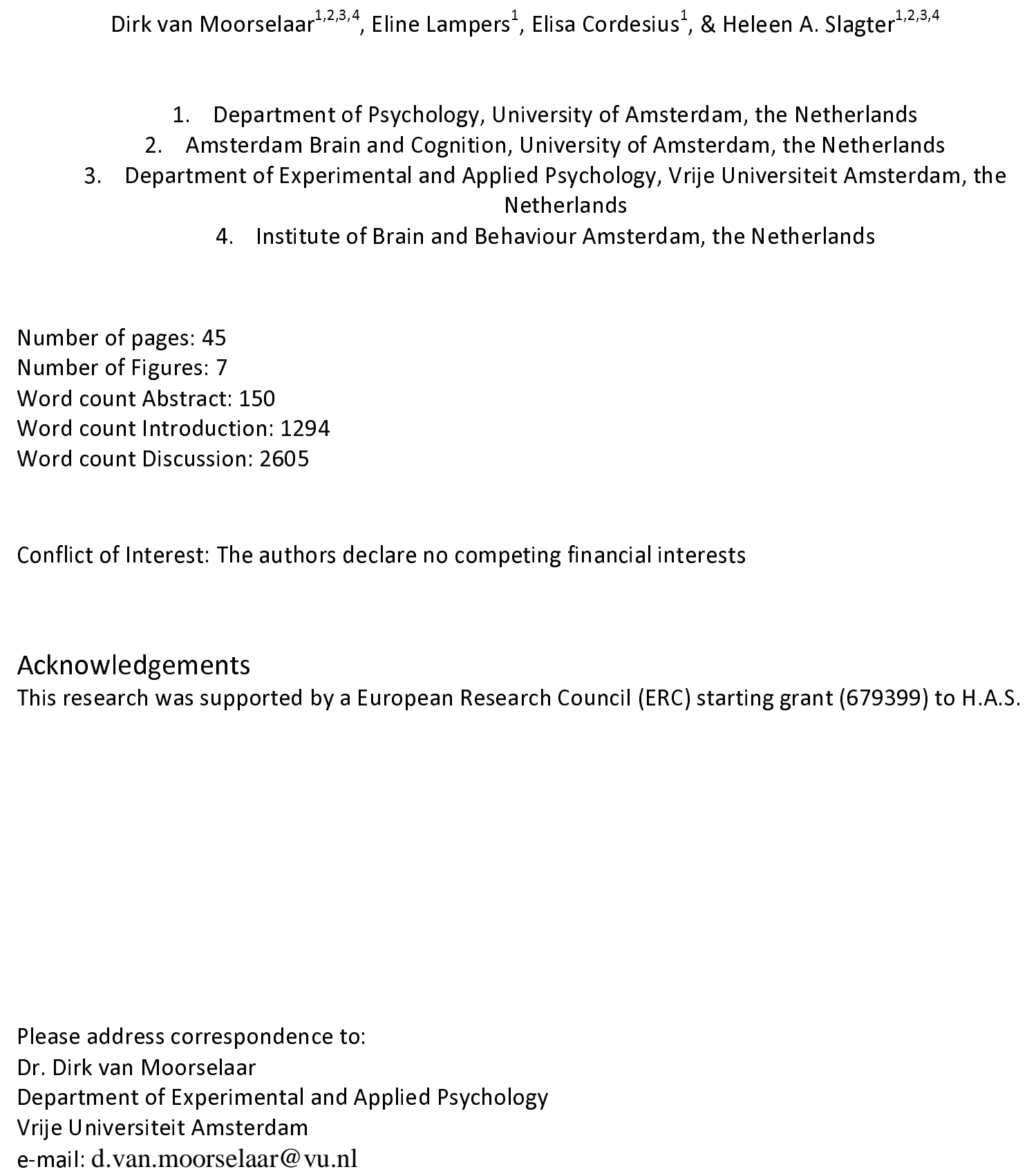




\section{Abstract}

49

50 Predictions based on learned statistical regularities in the visual world have been shown to facilitate

51 attention and goal-directed behavior by sharpening the sensory representation of goal-relevant

52 stimuli in advance. Yet, how the brain learns to ignore predictable goal-irrelevant or distracting

53 information is unclear. Here, we used EEG and a visual search task in which the predictability of a

54 distractor's location and/or spatial frequency was manipulated to determine how spatial and feature

55 distractor expectations are neurally implemented and reduce distractor interference. We find that

56 expected distractor features could not only be decoded pre-stimulus, but their representation

57 differed from the representation of that same feature when part of the target. Spatial distractor

58 expectations did not induce changes in preparatory neural activity, but a strongly reduced $\mathrm{Pd}$, an

59 ERP index of inhibition. These results demonstrate that neural effects of statistical learning critically

60 depend on the task relevance and dimension (spatial, feature) of predictions. 


\section{Introduction}

62

63 The ability to ignore distracting information is a key component of selective attention, and critical to

64 goal-directed behavior. Recent studies suggest that while attention can be flexibly directed at goal-

65 relevant information, suppression of distracting information is not under similar voluntary top-down

66 control (Chelazzi, Marini, Pascucci, \& Turatto, 2019; Noonan, Crittenden, Jensen, \& Stokes, 2018), as

67 was previously generally assumed (Jensen \& Mazaheri, 2010). Rather, this work shows that

68 distractor suppression strongly depends on distractor-based learning, for example, about its likely

69 location in the visual environment (Failing, Wang, \& Theeuwes, 2019; Ferrante et al., 2018; Goschy,

70 Bakos, Müller, \& Zehetleitner, 2014; Reder, Weber, Shang, \& Vanyukov, 2003; Sauter, Liesefeld,

71 Zehetleitner, \& Müller, 2018; Wang \& Theeuwes, 2018a) or its non-spatial features, e.g., its color or

72 shape (Cunningham \& Egeth, 2016; Stilwell, Bahle, \& Vecera, 2019; Vatterott \& Vecera, 2012). Based

73 on these observations, it has been proposed that distractor inhibition may be dependent on

74 expectations derived from past experience about the likelihood of events (Noonan et al., 2018; van

75 Moorselaar \& Slagter, 2020). This idea is grounded in recent notions of predictive processing

76 (Friston, 2009) in which the brain continuously uses statistical regularities in the environment to

predict the outside world with the overall aim of reducing the mismatch between its a priori predictions and the sensory input. In this framework, processing of any expected stimulus, whether relevant or irrelevant, is reduced (as it elicits a smaller prediction error). Distractor expectations should hence reduce subsequent distractor processing and interference. Yet, it remains unclear how learned inhibition, which often occurs implicitly (Ferrante et al., 2018; Wang \& Theeuwes, 2018a), is neurally implemented.

Many studies have shown that attention can bias sensory processing of expected taskrelevant (or target) information in advance, both on the basis of non-spatial feature (Battistoni, Stein, \& Peelen, 2017) and location information (Giesbrecht, Weissman, Woldorff, \& Mangun, 2006; Hopfinger, Buonocore, \& Mangun, 2000; Kok, Rahnev, Jehee, Lau, \& De Lange, 2012). Yet, it is currently unclear if expectations about upcoming distracting information can similarly prepare the 
system in advance by modifying activity in sensory regions representing the expected distractor

89 location or its non-spatial features, or alternatively, that distractor learning only becomes apparent

90 upon integration with incoming sensory information (van Moorselaar \& Slagter, 2020). Thus far, this

91 question has only been directly addressed in the context of spatial distractor expectations, with

92 mixed findings. One recent EEG study reported increased pre-stimulus alpha-band activity over

93 visual regions representing the likely distractor location, which was interpreted to reflect top-down

94 preparatory inhibition (Wang, van Driel, Ort, \& Theeuwes, 2019). In contrast, two other recent EEG

95 studies did not observe any learning-induced changes in preparatory activity of visual regions

96 representing the expected distractor location, as captured by pre-stimulus alpha-band activity

97 (Noonan et al., 2016) or by changes in anticipatory spatial tuning as revealed by inverted encoding modeling based on alpha-band activity (van Moorselaar \& Slagter, 2019). This discrepancy in findings

99 may be explained by the fact that in the former study by Wang et al. (2019) distractor learning could

100 only be location-based, as the shape and color of the distractor stimulus varied from trial to trial,

101 while in the latter two studies distractor features were fixed, also permitting build-up of feature-

102 based expectations. This may have led to stronger location-based learning in the Wang et al. (2019)

103 study. Indeed, in this study, it was also found that even targets occurring at the likely distractor

104 location were inhibited, as reflected by slower response times. Behavioral studies have also shown,

105 however, that when targets and distractors are assigned unique and fixed features, the suppression

106 at high probability distractor locations becomes specific to distractors (Allenmark, Zhang, Liesefeld,

107 Shi, \& Müller, 2019; Zhang, Allenmark, Liesefeld, Shi, \& Muller, 2019), indicative of feature-based

108 inhibition. Together, these findings suggest that the locus of distractor learning in the processing

109 hierarchy is flexible and depends on the extent to which expectations are location- and/or feature-

110 specific.

111 It is currently highly controversial whether expected distractor features can also be 112 represented as so called 'templates for rejection' (Woodman \& Luck, 2007). Although some 113 behavioral findings are in line with this idea (Arita, Carlisle, \& Woodman, 2012; Carlisle \& Nitka, 
114 2018; Park, Kim, \& Chun, 2007; Woodman \& Luck, 2007), to date there is no neural evidence for

115 feature-based distractor templates that set up the system in advance to inhibit distractor processing.

116 However, the few studies that investigated this, cued the distractor on a trial-by-trial basis (de Vries,

117 Savran, van Driel, \& Olivers, 2019; Reeder, Olivers, Hanke, \& Pollmann, 2018), preventing distractor

118 learning and leaving open the possibility that anticipatory distractor feature templates only arise

119 after repeated encounters with the to-be-ignored feature, i.e., when statistical learning is possible.

120 In the current study, we aimed to determine if and how location- and feature-based

121 distractor expectations may independently or in interaction bias sensory regions in advance to

122 suppress distractor processing using visual search tasks and EEG. Across visual searches the

123 distractor appeared more frequently on one location (i.e., high probability distractor location) than

124 on all other locations (i.e., low probability distractor locations). Critically, the target and distractor

125 either shared the same spatial frequency, or had different spatial frequencies, that could either be

126 predicted in advance or not (see Figure 1). This allowed us to investigate whether preparatory

127 suppression of activity in brain regions representing the high probability distractor location, as

128 reflected in pre-stimulus alpha-band activity, is dependent on expectations at the feature level. In

129 addition, using multivariate decoding, we could determine if learning about upcoming distractor

130 features (i.e., their spatial frequency) modulated activity of visual regions representing these

131 features in advance, suggestive of a template for rejection. 

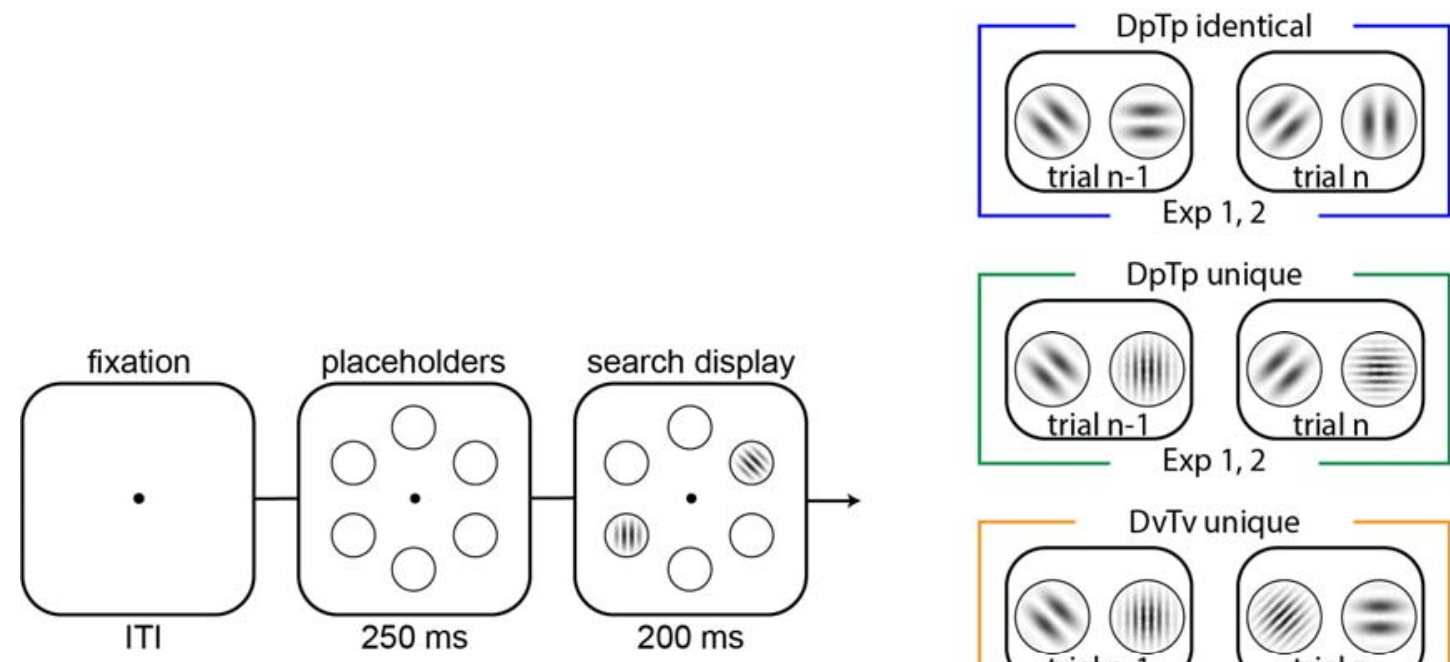

$\operatorname{Exp} 1,2$

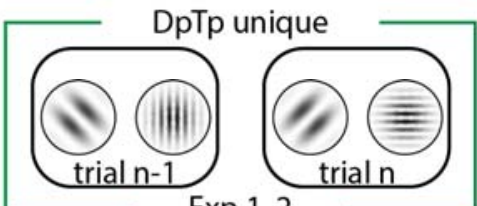

Exp 1,2

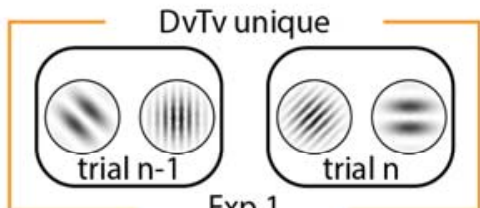

Exp 1

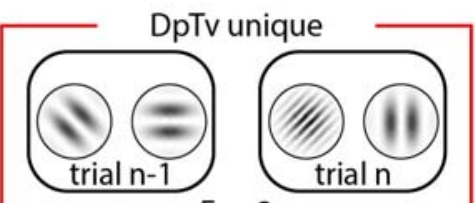

$\operatorname{Exp} 2$

133 Figure 1 | Task design of Experiments 1 and 2. Across conditions (colored boxes on the right), in each trial, participants had to indicate the orientation tilt (left or right) of a target gabor. In all conditions, a distractor (a gabor that was horizontally or vertically oriented) could be concurrently presented, which appeared with $70 \%$ probability on one specific location. The search display was presented for 200-ms, and participants had 1000ms to respond. In the DpTp identical and DpTP unique (Experiment 1 and 2) conditions, the spatial frequency of the target and distractor were fixed within an experimental block of trials, with targets and distractors having the same spatial frequency in the former and different spatial frequencies in the latter. In DvTv unique condition (Experiment 1), the target and distractor had different spatial frequencies, which were randomly selected across trials. Finally, in the DpTv condition (Experiment 2) the target and distractor had different spatial frequencies, but only the distractor spatial frequency was fixed across trials in an experimental block. The visualized spatial frequencies do not correspond to the frequencies used in the experiment. Note further

location and feature suppression, it is important to realize that the absence of such effects does not

necessarily imply that inhibition is purely reactive. It has recently been proposed that salient

distractors can be filtered out pre-attentively (Gaspelin \& Luck, 2018b; Sawaki \& Luck, 2010). This so-

150 called signal suppression hypothesis is largely based on the observation that in some contexts,

151 salient distractors can be suppressed, as indicated by a Pd, an ERP marker linked to inhibition, before

152 they capture attention, as indicated by the absence of a distractor-evoked N2pc (Burra \& Kerzel,

153 2013; Gaspar \& McDonald, 2014; Gaspelin \& Luck, 2018a; Jannati, Gaspar, \& McDonald, 2013; Wang 
154 et al., 2019). Intriguingly, we recently found that in a context that enforced learning at the spatial

155 level, as distractors and targets were very similar at the feature level (i.e., same spatial frequency),

156 distractors at expected locations continued to elicit an N2pc, but the subsequent Pd was virtually

157 eliminated. We interpreted this as if there was no more need for post-distractor inhibition once

158 participants had learned that information at a specific location could be safely ignored (van

159 Moorselaar \& Slagter, 2019). Here, we therefore also examined whether a similar pattern is

160 observed when spatial expectations can be combined with feature expectations, or whether

161 distractor-feature specific learning does allow for pre-attentive suppression.

162 In brief, our results demonstrate that expectations about features of upcoming distractors in

163 the environment induce pre-stimulus sensory templates. Crucially, these sensory templates differed

164 as a function of whether the feature was a predictable feature of the target or of the distractor,

165 providing evidence for distinct target and distractor templates. Yet, we find no evidence for a

166 preparatory spatial bias as a function of distractor location predictability, as no modulations of pre-

167 stimulus alpha-band activity were observed. Distractors at expected locations did evoke a smaller

$168 \mathrm{Pd}$, indicative of a reduced need for inhibition, independent of distractor feature expectations. Thus,

169 neural effects of predictions based on regularities in the environment critically depend not only on

170 the task relevance (target, distractor), but also the dimension (spatial, feature) of these regularities.

171

172

173 We conducted two experiments, a behavioral and EEG experiment. The methods and analyses of 174 both these experiments were preregistered at the Open Science Framework (https://osf.io/7ek45/).

176 Experiment 1 - Is spatial suppression modulated by feature expectations?

177 We first conducted a behavioral experiment to examine how distractor expectations at the feature 178 and spatial level may interact in reducing distractor interference. We specifically predicted that 179

\section{Results}

\author{
and spatial level may interact in reducing distractor interference. We specifically predicted that
} suppression at high probability distractor locations would become specific to distractors (i.e., not 
180 affect target processing) when distractors are assigned unique and fixed spatial frequencies, as has

181 been shown previously using shapes and colors (Allenmark et al., 2019; Zhang et al., 2019). To this

182 end, observers $(N=18)$ performed a visual search task in which the distractor and the target either

183 had the same spatial frequency and only differed in orientation (referred to as Distractor predictable

184 Target predictable (DpTp) identical condition), or differed both in spatial frequency and orientation.

185 In this latter scenario, target and distractor spatial frequencies were either selected at random

186 (referred to as Distractor variable Target variable (DvTv) unique condition) or fixed across a

187 sequence of trials (referred to as DpTp unique condition). This allowed us to test whether learning

188 selectively occurs at the spatial level when search is necessarily feature non-specific, either because

189 target and distractors share the same spatial frequency or because their spatial frequency varies

190 randomly, but not when distractor features can be predicted in advance during feature-specific

191 search (see Figure 1).

\section{Behavior}

194 Figure 2A (left plot) shows the mean reaction times (RTs) for the search task as a function of the 195 distractor location for all conditions. These measures were entered into a two-way repeated 196 measures ANOVA ( $N=18$ ) with the within-subjects factors Condition (DpTp identical, DvTv unique,

197 DpTp unique) and Distractor Location (high probability distractor location, low probability distractor 198 location). Replicating previous findings (e.g.,)(Ferrante et al., 2018; Wang \& Theeuwes, 2018a), RTs

199 were faster when the distractor appeared at high relative to low probability distractor locations

200 (main effect Distractor location; $F(1,17)=97.0, p<0.001, \eta_{p}^{2}=0.85$ ). Moreover, there was a main

201 effect of Condition $\left(F(2,34)=15.8, p<0.001, \eta_{p}^{2}=0.48\right)$ reflecting faster RTs in the DpTp unique

202 condition, in which spatial frequencies of both the target and distractor could be anticipated,

203 relative to conditions in which those spatial frequencies could not guide search, either because the

204 target and distractor spatial frequency were identical or because they varied from trial to trial.

205 Critically, a significant interaction $\left(F(2,34)=5.2, p=0.011, \eta_{p}^{2}=0.23\right)$ showed that the RT benefit at 
206 high probability distractor locations was modulated by the extent to which distractor- and target-

207 specific predictions could be formed at the feature level. Planned comparisons revealed that while

208 distractors were more efficiently ignored on high probability distractor locations across all conditions

209 (all $t^{\prime} s>6.7$, all $p^{\prime} s<0.001$, all $d^{\prime} s>1.58$ ), RTs were reduced most by distractor location predictability

210 when distractors and targets could not be dissociated at the feature level in advance. Specifically, in

211 feature specific search (i.e., DpTp unique) spatial benefits were smaller than during feature non-

212 specific searches (DpTp unique vs. DpTp identical, $t(17)=3.0, p=0.008, d=0.71$; DpTp unique vs.

213 DvTv unique, $\left.t(17)=2.0, p=0.06, d=0.48 ; B F_{10}=1.3\right)$, whereas spatial benefits did not differ

214 between the two feature non-specific search conditions (DpTp identical vs. DvTv unique, $t(17)=1.3$,

$215 p=0.22, d=0.30 ; B F_{01}=2.1$ ). These findings are indicative of stronger suppression at the high

216 probability distractor location in the condition in which expectations could necessarily only develop

217 at the spatial level. 
A

Distractor location tuned

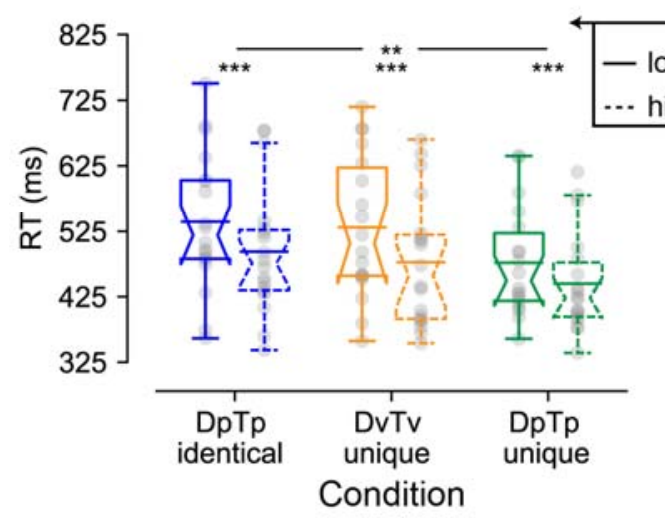

B

\section{Target location tuned}

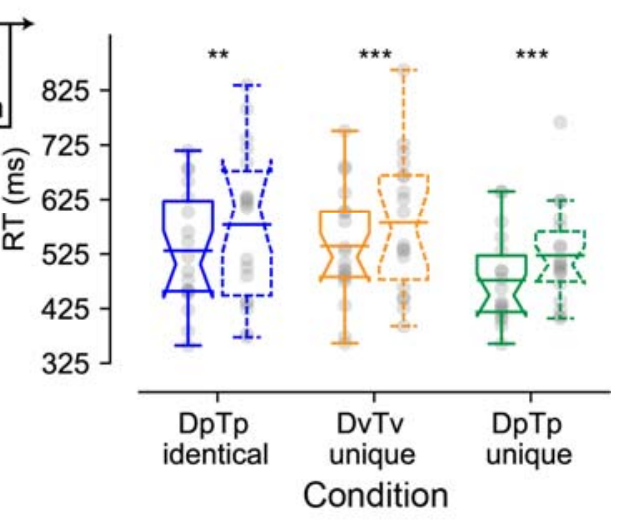

Distractor location tuned

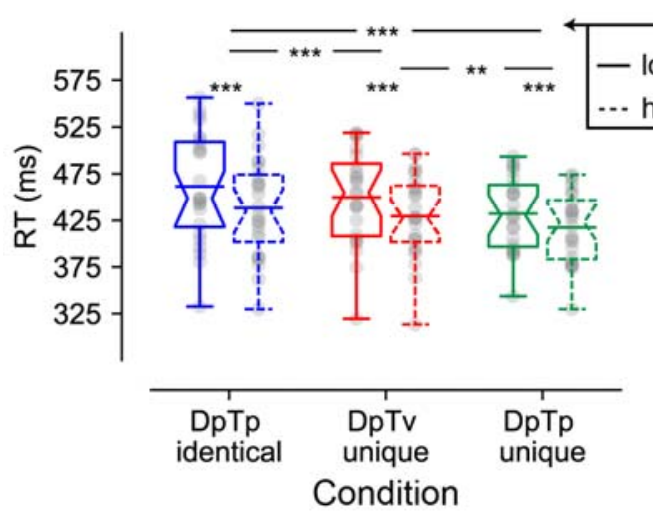

Target location tuned

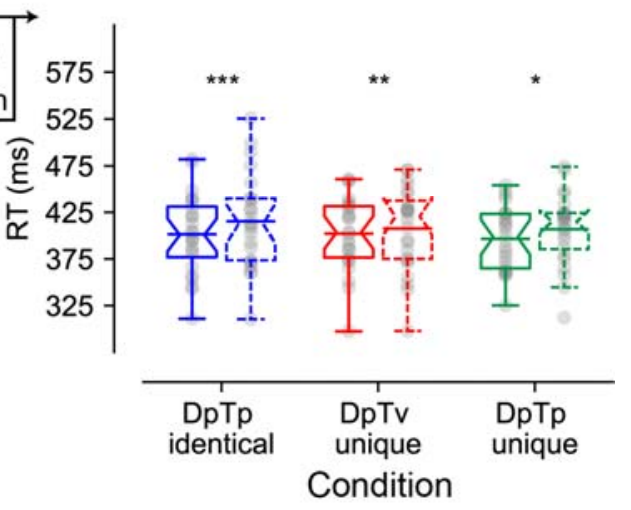

Figure 2 | Behavioral findings of Experiment 1 (A) and 2 (B) visualized by notched boxplots with solid horizontal lines corresponding to the mean. (A) Reaction times as a function of distractor location (left) and target location (right) in Experiment 1. (B) Reaction times as a function of distractor location (left) and target location (right) in Experiment 2, where the target location tuned plot only is based only on distractor absent trials. All distractor location tuned plots do not contain trials with the target at the high probability location, and the target location tuned plots do not contain distractors at high probability locations. DPTp indentical: the distractor and the target had the same predictable spatial frequency (fixed across a sequence of trials); DVTV unique: targets and distractors had different, but unpredictable spatial frequencies (varied across a sequence of trials). DpTp unique: targets and distractors had different and predictable spatial frequencies (fixed across a sequence of trials). DpTv unique: targets and distractors had different spatial frequencies, but only the distractor spatial frequency was predictable (fixed across a sequence of trials).

230

To ensure that the observed reductions in distractor interference at high probability distractor locations do not merely reflect intertrial location priming (Maljkovic \& Nakayama, 1994), we repeated the preceding analysis after excluding all trials in which the distractor location repeated. This control analysis mimicked the main findings, although the main effects of Condition and Distractor Location (all $F^{\prime} s>16.4$, all $p^{\prime} s<0.001$, all $\eta_{p}^{2}$ 's $>0.49$ ) were no longer accompanied by 
237 a significant interaction $\left(F(2,34)=1.9, p=0.17, \eta_{p}^{2}=0.10 ; B F_{01}=4.9\right)$. Importantly, however, across

238 all conditions distractors were more efficiently ignored on high probability distractor locations (all $t^{\prime} s$

$239>2.6$, all $p^{\prime} s<0.018$, all $d^{\prime} s>0.62$ ) demonstrating that intertrial location priming could not entirely

240 account for the observed reduction in distractor interference at the high probability distractor

241 location, as also reported in previous studies (e.g.))(Failing, Feldmann-Wustefeld, Wang, Christian, \&

242 Theeuwes, 2019; Ferrante et al., 2018).

243 To examine whether the efficiency of target selection was also affected by distractor

244 location and/or feature foreknowledge, we repeated the same analysis but now tuned to the target

245 location (see Figure 2A; right). Consistent with generic suppression at the high probability distractor

246 location, target processing was also impaired at high probability distractor locations (main effect

247 Distractor location; $\left.F(1,17)=28.2, p<0.001, \eta_{p}^{2}=0.62\right)$. However, this impairment did not differ

248 between conditions (Condition by Distractor Location interaction: $F(2,34)=0.09, p=0.92$,

$\left.249 \eta_{p}^{2}=0.005 ; B F_{01}=6.7\right)$ suggesting that processing of any target was suppressed to the same extent

250 at the high probability distractor location independent of its similarity to the distractor at the feature

251 level or whether participants had advance knowledge about its spatial frequency.

To summarize, Experiment 1 showed that 1) distractor interference is most reduced when

distractors can be predicted at both the spatial and feature level; 1 ) distractor location learning is

more pronounced when distractors can only be predicted on the basis of location, but not feature

information; and that 3 ) any stimulus presented at the expected distractor location is automatically

inhibited, including targets. Specifically, target processing at the high probability distractor location

was slowed uniformly across conditions (independent of feature expectations), while the effects of

260 of suppression tied to the high probability location was fixed, subsequent inhibition of stimuli at the

261 likely distractor location is modulated by expectations about what search items look like at the

262 feature level. It should be noted however that the observed interaction between spatial and feature 
263 expectations in the distractor-tuned analysis disappeared when we controlled for intertrial spatial

264 priming, an aspect that we will revisit in Experiment 2, which contained many more trials per

265 condition, and hence permitted for the development of more robust spatial and feature

266 expectations.

267

268 Experiment 2: Neural mechanisms underlying learned distractor suppression

269 The findings from Experiment 1 suggest that the level of the processing hierarchy at which distractor

270 expectations are implemented is dependent on whether expectations can only be developed on the

271 basis of spatial information or can also be grounded in non-spatial regularities. In Experiment 2, we

272 used EEG and a visual search task in which we again manipulated the predictability of a distractor's

273 location and/or spatial frequency to determine how spatial and feature distractor expectations are

274 neurally implemented and may interact in reducing distractor interference. The visual search task

275 was similar to the task used in Experiment 1 with two notable exceptions. In Experiment 1, we found

276 that distractor inference was weakest when both the location and feature of the distractor were

277 predictable. Yet, in this condition (DpTp unique), targets were also predictable at the feature level,

278 rendering it possible that part of this effect is due to target feature expectations. As we were

279 specifically interested in the interaction between distractor learning at the feature and the spatial

280 level and since Experiment 1 showed no difference between the conditions with identical (DpTp

281 identical) and randomly alternating (DvTv unique) spatial frequencies (supposedly both enforcing

282 spatial learning), we replaced the latter condition by a condition in which only the distractor feature

283 was fixed and therefore predictable (DpTv unique). In this condition, the spatial frequency of the

284 target varied from trial to trial and was always different from the fixed and thereby predictable

285 spatial frequency of the distractor. Also, in Experiment 2, we included distractor absent trials, which

286 allowed us to both quantify the magnitude of distractor interference across conditions and get a

287 better estimate of how target processing was affected at predicted distractor locations. 
288 The goal of Experiment $2(N=24)$ was threefold. First, we aimed to establish whether a

289 preparatory alpha gating mechanism contributes to inhibition of the activity of brain regions

290 representing the expected distractor location, and if so, whether such preparatory spatial inhibition

291 is more pronounced in, or even specific to situations in which learning cannot take place at the

292 feature level. Note that using a similar set-up, we recently did not observe any changes in

293 preparatory alpha-band tuning to the expected distractor location (van Moorselaar \& Slagter, 2019).

294 However, in that study, the distractor's location was repeated across four consecutive trials only,

295 leaving open the possibility that there was simply insufficient time for location predictions to

296 develop. Secondly, we aimed to determine whether preparatory neural activity (also) contains

297 information about the expected feature of the upcoming distractor, and if this is dependent on

298 whether the target is also predictable at the feature level. As noted above, the notion of templates

299 for rejection is currently highly contested. Finally, we aimed to extend our recent finding that

300 distractor location expectations reduced post-distractor inhibition, as indicated by a greatly reduced

301 or even eliminated Pd ERP (van Moorselaar \& Slagter, 2019), by determining if this effect is also

302 dependent on feature-based distractor expectations or not.

305 Figure 2B shows the mean reaction times (RTs) for the search task as a function of the distractor

306 location for all conditions, which were analyzed with a two-way repeated measures ANOVA with the

307 within-subjects factors Condition (DpTp identical, DpTv unique, DpTp unique) and Distractor

308 Location (high probability distractor location, low probability distractor location). As in Experiment 1,

309 distractors at high probability distractor locations interfered to a lesser extent with target search

310 than distractors at low probability distractor locations (main effect Distractor Location; $F(1,23)=$

311 149.0, $p<0.001, \eta_{p}^{2}=0.87$ ), although RTs at high probability distractor locations were still slower

312 compared to distractor absent trials (all $t^{\prime} s>9.2$, all $p^{\prime} s<0.001$, all $d^{\prime} s>1.87$ ). As reflected by a main

313 effect of Condition $\left(F(2,46)=17.5, p<0.001, \eta_{p}^{2}=0.43\right)$, overall RTs were fastest when both the 
314 target and distractor spatial frequency were predictable and could guide search, intermediate when

315 only the distractor's spatial frequency could guide search and slowest when the spatial frequency of

316 target's and distractors was identical and hence could not guide search. Importantly, and replicating

317 Experiment 1, while distractor interference was reliably reduced at high probability distractor

318 locations across all conditions (all $t^{\prime} s>10.1$, all $p^{\prime} s<0.001$, all $d^{\prime} s>2.07$ ), this benefit decreased the

319 more visual search could rely of feature expectations, as captured by a significant Distractor Location

320 by Condition interaction $\left(F(1,32)=16.5, p<0.001, \eta_{p}^{2}=0.42\right)$. Indeed, the benefit of distractor

321 location predictability (i.e., high vs. low) was most pronounced when distractors and targets

322 consistently shared the same spatial frequency and learning could only be location-based $(M=50.9$

$323 \pm S D=24.4$; DpTp identical vs. DpTp unique: $t(23)=4.6, p<0.001, d=0.93$; DpTp identical vs. DpTv

324 unique; $t(23)=4.1, p<0.001, d=0.84)$, intermediate when feature prediction could be formulated

325 only for the distractor $(M=41.7 \pm S D=20.1)$ and smallest when feature predictions could be

326 formulated for both the distractor and target $(M=30.6 \pm S D=10.7$; DpTv unique vs. DpTp unique: $t$

$327(23)=3.0, p=0.005, d=0.62$ ). These results suggest that distractor feature learning may shift the

328 locus in the visual hierarchy at which expectation-based suppression operates from space-based to

329 feature-based.

330 Next, we again examined whether the observed reductions in distractor interference at high

331 probability distractor locations were the consequence of statistical learning rather than simply of

332 short-lasting, inter-trial location priming (Maljkovic \& Nakayama, 1994). After exclusion of all trials in

333 which the distractor location repeated between trials, we again observed main effects of Distractor

334 Location and Condition (all $F^{\prime} s>19.7$, all $p^{\prime} s<0.001$, all $\eta_{p}^{2}{ }^{\prime} s>0.46$ ) and, counter to Experiment 1 ,

335 the interaction also remained reliable $\left(F(1,32)=9.6, p<0.001, \eta_{p}^{2}=0.29\right)$, indicating that neither

336 the effect of distractor location predictability on visual search times, nor the differences between

337 conditions therein could be simply explained by intertrial spatial priming effects.

338 Having established that distractors were generally more efficiently ignored at high

339 probability distractor locations, we next investigated whether as in Experiment 1, target processing 
340 was impaired at high probability distractor locations and if this was dependent on the extent to

341 which targets and/or distractors were predictable at the feature level. For this purpose, we repeated

342 the preceding analyses but only included distractor absent trials. As visualized in Figure 2B (right)

343 and consistent with Experiment 1, target detection was hampered at high probability distractor

344 locations across conditions (main effect Target Location; $F(1,23)=25.4, p<0.001, \eta_{p}^{2}=0.53$ ). As in

345 Experiment 1, this effect was not accompanied by an interaction with Condition suggesting that

346 target processing was impaired to the same extent at high probability distractor locations

347 irrespective of the nature of feature expectations $\left(F(2,46)=1.8, p=0.18, \eta_{p}^{2}=0.071 ; B F=0.07\right)$.

348 Together, these results replicate the finding of Experiment 1 that the visual system 349 continues to be sensitive to feature information at a location that is suppressed in advance (Stilwell 350 et al., 2019). Whereas target processing was impaired to the same extent across conditions, arguing 351 for a fixed level of suppression tied to the high probability location, subsequent inhibition of 352 distracting stimuli at this location was modulated by expectations at the feature level, with 353 suppression being most pronounced when learning could necessarily only be space-based. In

354 Experiment 2, this interaction between spatial and feature expectations remained significant even 355 after controlling for spatial intertrial transitions, suggesting that when expectations can be grounded 356 in longer learning, the system combines spatial and feature information about probable upcoming 357 distractors to reduce distractor interference. This makes sense from an ecological perspective: one 358 would not want to simply inhibit anything occurring at a particular location in space.

EEG

Time-frequency analyses: Pre-stimulus alpha-band activity does not increase contralateral to the high probability distractor location

365 At the behavioral level, we found that spatial distractor expectations generally reduced distractor

366 interference during visual search, albeit most strongly so in the absence of distractor feature expectations, and furthermore, that target search was slowed down when the target occurred on 
368 the high probably distractor location, indicative of generic spatial suppression. Using time-frequency

369 analyses, we first examined if location-based distractor expectations modulated activity of visual

370 regions representing the likely distractor location in advance, as indexed by a pre-stimulus alpha-

371 band lateralization index at posterior electrode sides (POS), where contralateral and ipsilateral were

372 relative to the high probability distractor location:

$$
\frac{a_{\text {contralateral POS }}-a_{\text {ipsilateral POS }}}{a_{\text {contralaeral POS }}+a_{\text {ipsilateral POS }}}
$$

373 and if so, whether pre-stimulus alpha-band lateralization reduced when learning could also occur at

374 the feature level. As we were specifically interested in lateralized alpha power as a measure of

375 anticipatory spatial suppression, we limited the statistical analyses to the anticipatory time window

376 (i.e., $-750 \mathrm{~ms}-0 \mathrm{~ms}$ ). Consequently, the analysis was only sensitive to learned expectations and

377 hence could not be confounded by the actual stimulus configuration, which allowed us to include all

378 trials. The lateralization index, which was analyzed at electrodes PO7/8 and $01 / 2$, has the benefit of

379 not having to select a pre-stimulus baseline window, the same time window where we expected

380 alpha-band modulations. Note, that for the same reason, Wang et al. (2019) developed a Z-

381 transformation procedure, which we (as preregistered) also applied here. This resulted in virtually

382 identical results as obtained with the lateralization index.

383 As visualized in Figure 3A/B, cluster-based permutation tests across time and frequency and

384 across time for lateralization index averaged within the alpha-band $(8-12 \mathrm{~Hz})$ did not show any

385 reliable increase of alpha-power contralateral vs. ipsilateral to the high probability distractor

386 location in any condition. Nevertheless, Figure 3B also suggests that in the DpTp identical condition -

387 the one condition in which learning could only take place at the location level, but not at the feature

388 level - a contralateral increase in alpha-band power was present numerically, albeit not significantly

389 so. To increase sensitivity to a potentially weak effect, in an exploratory analysis, we entered the

$390 \quad a$ lateralization index averaged over the entire anticipatory time window into a repeated measures

391 ANOVA. Critically, this analysis did not show a main effect of Condition $(F(2,46)=0.4, p=0.68$, 
393 condition $\left(t(23)=1.2, p=0.21, d=0.27 ; B F_{01}=2.22\right)$. Thus, these time-frequency findings suggest

394 that the observed spatial suppression at the high probability distractor location at the behavioral

395 level was not mediated by a contralateral increase of alpha-band power in anticipation of search

396 display onset, even in a condition in which learning could only occur at the spatial level and across a

397 longer time span than in two previous studies that also did not observe distractor learning-related

398 changes in pre-stimulus alpha-band activity (Noonan et al., 2016; van Moorselaar \& Slagter, 2019),

399 but in contrast to another study (Wang et al., 2019).

A

DpTp identical

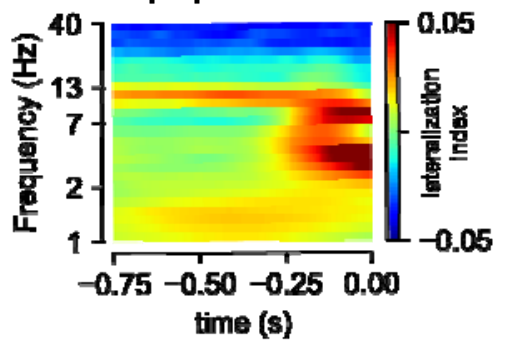

B

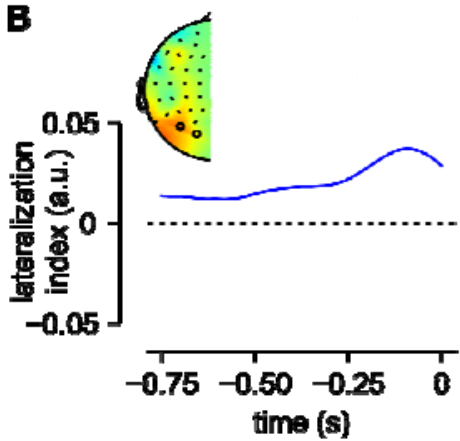

DpTv unique
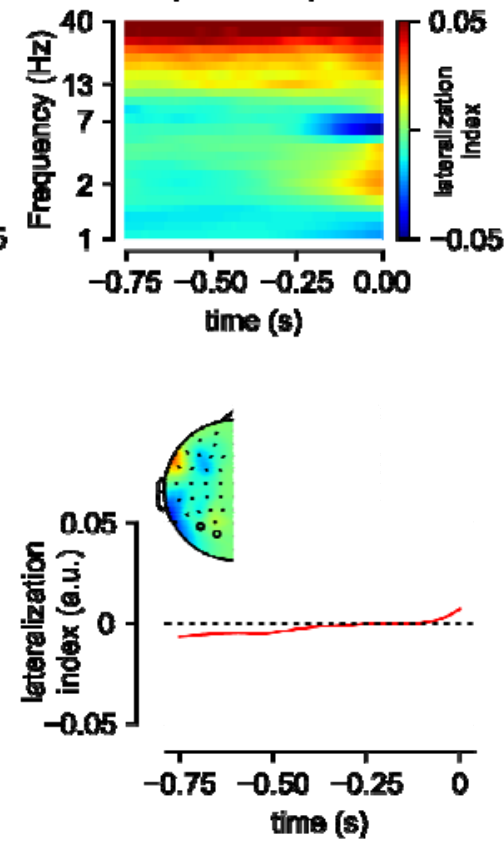

DpTp unique
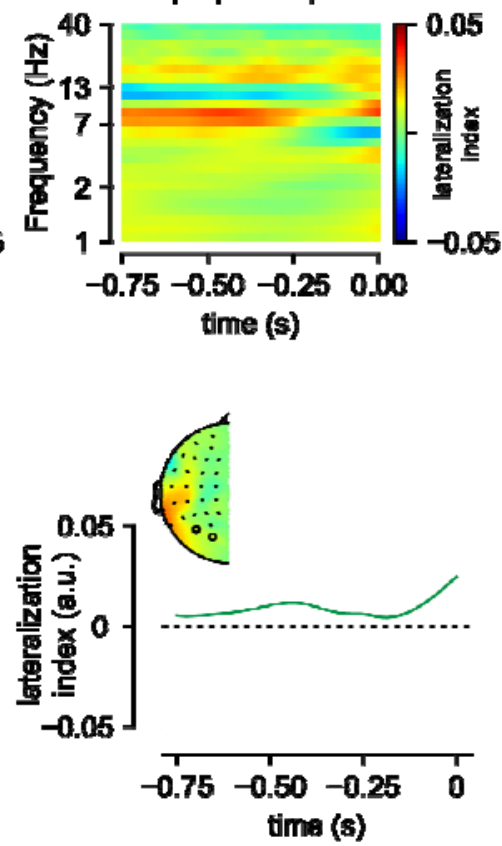

400

401

402

403

404

405

406

407

408

409

410

411

412

413

Figure 3 | Anticipatory alpha-activity. (A) These panels show the lateralization index relative to the high probability distractor location averaged over PO7/8 and 01/2 separately per condition. (B) Time-series of lateralization index averaged within the alpha-band $(8-12 \mathrm{~Hz})$. Insets show topographic distribution averaged over the entire anticipatory window. Although numerically, pre-stimulus alpha-band activity was higher over visual regions contralateral to the predicted distractor location in the DpTp indentical condition in which the distractor and the target had the same fixed spatial frequency, permitting only the development of spatial distractor expectations, this effect was not statistically significant. DpTv unique: targets and distractors had different spatial frequencies, but only the distractor spatial frequency was predictable (fixed across a sequence of trials); DpTp unique: targets and distractors had different and predictable spatial frequencies (fixed across a sequence of trials).

Decoding: Target and distractor-based feature expectations induce pre-stimulus sensory templates 
415 Our behavioral findings showed that distractor feature-specific expectations in the absence of any

416 target expectations modulated spatial expectation-dependent suppression, suggesting that non-

417 spatial information about upcoming distractors reduced distractor interference. To investigate

418 whether feature-specific distractor expectations induced pre-stimulus sensory templates, i.e., were

419 already evident in the anticipatory EEG signal, we trained a classifier on the response pattern of 64

420 electrodes using each spatial frequency (i.e., low, medium, high) for each timepoint, separately for

421 targets and distractors (see Methods section for details). ERP studies have demonstrated differences

422 in early visual-evoked responses as a function of stimulus spatial frequency (e.g.,) (Kenemans, Kok, \&

423 Smulders, 1993; Proverbio, Zani, \& Avella, 1996), an effect that is attributed to macro-scale

424 differences in sensitivity to spatial frequency across visual regions (Kenemans, Baas, Mangun, Lijffijt,

$425 \&$ Verbaten, 2000). Therefore, one would expect that multivariate pattern analysis techniques are

426 also sensitive to spatial frequency as measured with EEG. Yet, to our knowledge, no study has so far

427 shown that it is also possible to decode spatial frequency from the pattern of scalp-EEG activity.

428 Classification performance visualized in Figure 4 shows that the classifier could discriminate

429 not only the target, but also the distractor's spatial frequency, in all conditions ${ }^{1}$. Interestingly, both

430 in anticipation and during stimulus processing, target decoding was numerically higher when its

431 spatial frequency was predictable (i.e., DpTp unique condition) compared to when it was not (i.e.,

432 DpTv unique condition), whereas the opposite pattern was observed for distractor decoding: the

433 spatial frequency of the distractor could be decoded with higher accuracy when only the spatial

434 frequency of the distractor, but not the target spatial frequency could be predicted in advance (in

435 the DpTv unique compared to the DpTp unique condition). The observation that the anticipatory

436 EEG signal already appeared to contain information about the upcoming distractor is especially

437 interesting as it may support the notion of a negative sensory template or preparatory feature-

\footnotetext{
${ }^{1}$ As it is unclear whether in the DpTp identical condition, in which targets and distractors were of the same spatial frequency, classification was driven by the spatial frequency of the target, of the distractor or both, we did not contrast this condition with the unique feature conditions.
} 
438 specific distractor inhibition. Critically, anticipatory target decoding was absent in the DpTv unique

439 condition, the one condition where target expectations could not develop, arguing against the

440 possibility that the observed anticipatory decoding can be explained by an artificial shift of the

441 stimulus-evoked response (van Driel, Olivers, \& Fahrenfort, 2019). Also note that anticipatory above

442 chance decoding was not sustained throughout the entire pre-stimulus period, but only emerged

443 close to search display onset, raising the possibility suggesting that sustained anticipatory

444 expectation signals were subtracted out by the baselining procedure (see methods). Control

445 analyses, however, using either a condition-specific pre-stimulus baseline (collapsed across spatial

446 frequency) or no baseline at all, yielded identical results indicating that the observed feature

447 specificity in the EEG signal only emerged in close temporal proximity to search display onset.

448 Cluster-based permutation tests across time confirmed this pattern of observations. As we

449 were specifically interested in anticipatory markers of distractor feature expectations, these tests

450 were performed separately for the anticipatory $(-750-0 \mathrm{~ms})$ and the reactive $(0-550 \mathrm{~ms})$ time

451 windows. As visualized by the red and green colored horizontal lines in Figure $4 \mathrm{~A}(p<0.05$, cluster-

452 corrected), anticipatory target decoding was specific to the DpTp unique condition, whereas

453 anticipatory distractor decoding was specific to the DpTv unique condition. These condition

454 differences were confirmed by a direct contrast between DpTp and DpTv unique conditions, which

455 yielded anticipatory and reactive differences between conditions, both for target and distractor

456 decoding ( $p<0.05$, cluster-corrected; double-colored lines in Figure 4A). These statistical results

457 reveal that when both the targets and the distractors spatial frequency could be predicted,

458 participants especially relied on target feature-specific information to guide search. However, when

459 they could not know what the target would look like, as it randomly varied across trials, and the

460 target should thus also not be evident in the anticipatory neural code, participants relied more on

461 distractor feature-specific information to bias search, and importantly, already in anticipation of

462 search display onset. 
A

Target decoding

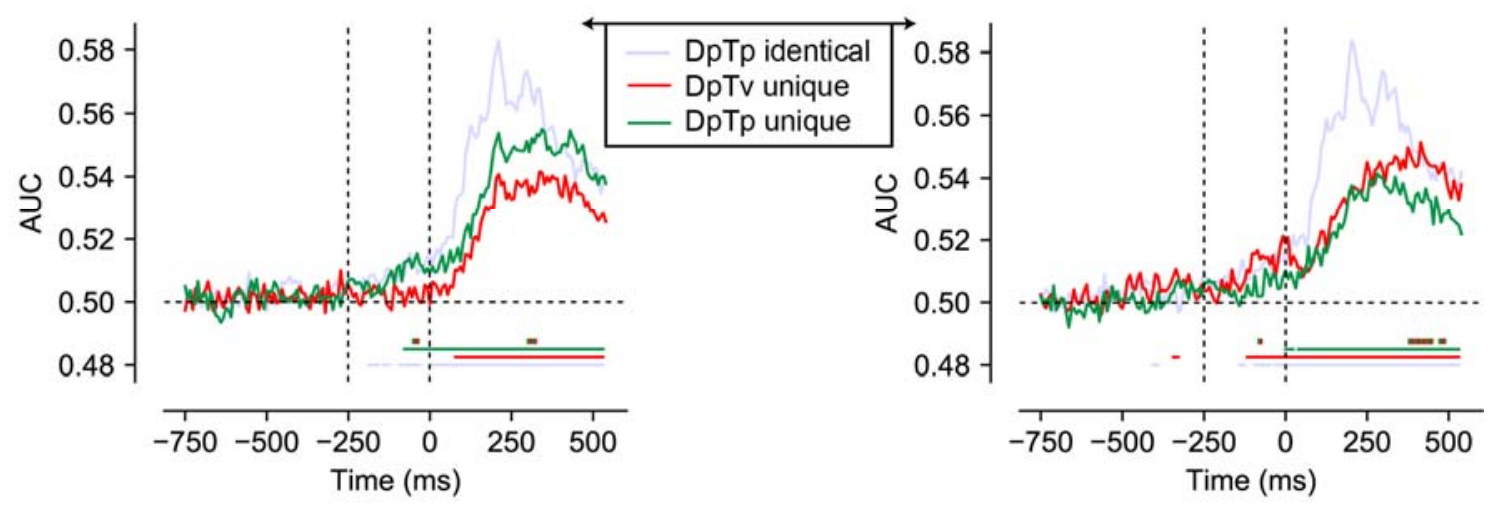

B

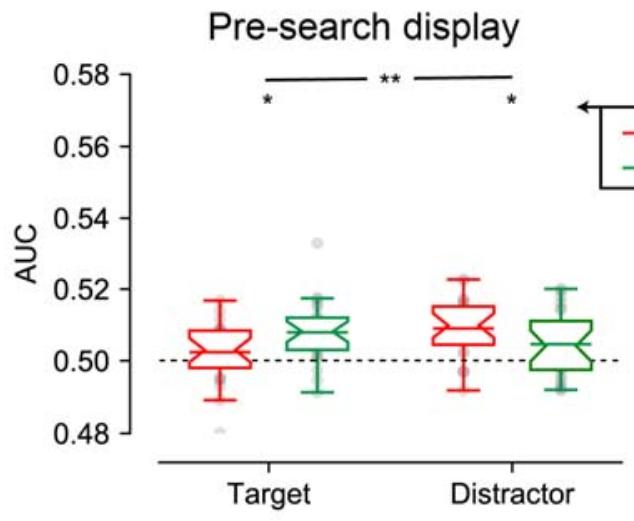

Stimulus class
Post-search display

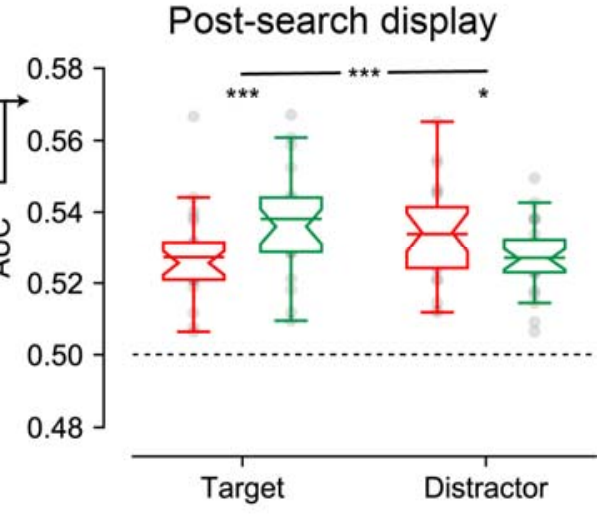

Stimulus class
465

466

467

468

469

470

471

472

473

474

475

476

Figure 4 | Multivariate decoding of the target and distractor spatial frequency across conditions using broadband EEG of all 64 electrodes. (A) AUC scores of target (left) and distractor (right) decoding across time. Colored bars on the $x$-axis (blue; red; green) indicate clusters where conditions differ significantly from chance after cluster correction ( $p<0.05$ ). Red and green dashed lines indicate clusters with a significant difference between DpTv unique and DpTp unique conditions after cluster correction $(p<0.05)$. (B) Averaged auc scores during the pre_search display (-250-0 ms; left) and the post-search display ( $0-550 \mathrm{~ms}$; right) visualized by notched boxplots with solid horizontal lines corresponding to the mean. Horizontal black dashed lines across plots indicates chance performance, whereas the vertical dashed black lines in the upper plots indicate the onset of placeholder $(-250 \mathrm{~ms})$ and the search display (Oms).

To more directly characterize these effects, we followed up with an exploratory analysis in which average decoding performance in separate time windows (pre-search display: $-250 \mathrm{~ms}$ to 0ms; or post-search display: $0 \mathrm{~ms}$ to $550 \mathrm{~ms}$ ) was used as the dependent variable in a repeated measures ANOVA with within-subject factors Stimulus Class (target, distractor) and Condition (DpTp unique, DpTv unique). As visualized in Figure 4B, and consistent with the pattern described thus far, this revealed significant effects of being able to predict the target and distractor spatial frequency in 
481 advance, that differed as a function of condition, both before the onset of the search display (in the

482 preparatory period) and after search display onset, as revealed by significant interactions between

483 Stimulus Class and Condition $\left(F(1,23)=11.3, p=0.003, \eta_{p}^{2}=0.33\right.$ and $F(1,23)=59.3, p<0.001$,

$484 \eta_{p}^{2}=0.72$, respectively). These interactions reflected better target decoding in the DpTp unique

485 than in the DpTv unique condition both before and after target presentation $(t(23)=2.1, p=0.044$,

$486 d=0.44$ and $t(23)=4.0, p<0.001, d=0.82$ respectively) and vice versa for distractor decoding $(t$

$487(23)=2.1, p=0.046, d=0.43$ and $t(23)=2.5, p=0.02, d=0.51$, respectively). Together, these

488 findings show that we could not only decode the spatial frequency of the target from the pattern of

489 pre-search display brain activity, but also, when the targets spatial frequency was unpredictable, the

490 spatial frequency of the distractor, suggestive of a distractor feature template

The observed anticipatory distractor decoding is in line with the idea that distractor-specific expectations may help to filter out distractors preemptively. Yet, it leaves open the question how they may do so, as decoding by itself is uninformative about the nature of the underlying neural representation (van Moorselaar \& Slagter, 2020). A hallmark of an advance rejection template is that processing of the coded features is inhibited and the neural representation of a rejection template should thus be distinct from a target-defined attentional template which in contrast facilitates processing of matching stimuli.

To test this prediction, we explored whether the pattern driving anticipatory distractor

503 decoding differed from that driving target decoding. Specifically, we trained the classifier separately

504 on either target or distractor spatial frequencies using the same trials that were used in the

505 preceding analyses. However, we now used the resulting weights to classify the spatial frequency of

506 the target in an independent set of trials, namely target-only trials without a distractor. The logic

507 here is that if targets and distractors are represented via the same neural code, it should not matter 
508 which of the two is used in the training stage. Yet, distractor weights should not generalize to targets

509 when they are represented via distinct neural codes.

510 As visualized in Figure 5A (solid lines) and as one would expect, the target trained model

511 tested on target-only trials replicated the findings of the main analysis: the spatial frequency of the

512 target could be reliably decoded post search display in all conditions, but also in anticipation of the

513 search display selectively in the condition in which the spatial frequency of the target was

514 predictable (DpTp unique; right plot). Cluster-based permutation tests across time confirmed that

515 target-target decoding was not only reliable in both the pre- and post-stimulus window $(p<0.05$,

516 cluster-corrected; solid green horizontal lines), but also differed from distractor-target decoding ( $p<$

5170.05 , cluster-corrected; black horizontal lines). Indeed, using distractor trained weights, the exact

518 same spatial frequency could no longer be decoded above change in advance when it was a

519 predictable feature of the target, potentially indicative of a distinct distractor template (Fig. 5A;

520 dashed lines). That is, the predictable target spatial frequency could only be decoded from the

521 pattern of pre-stimulus EEG activity using target-based classification weights, but not using

522 distractor-based classification weights, even though this same spatial frequency could be

523 reconstructed using distractor-based decoding (Figure 4). What's more, pre-stimulus target decoding

524 performance of the distractor-trained model did not differ between the conditions in which the

525 target spatial frequency could be predicted in advance vs. could not be predicted in advance, neither

526 when tested with cluster correction nor with paired t-tests within the pre-search display window $(t$

$\left.527(23)=0.57, p=0.57, d=0.12 ; B F_{01}=4.03\right)$ or the post-search display window $(t(23)=0.14, p=0.89$,

$528 d=0.028 ; B F_{01}=4.61$ ). Nonetheless, the Stimulus Class (target trained, distractor trained) by

529 Condition interaction was not significant in the pre-search display window $(F(1,23)=0.6, p=0.44$,

$530 \quad \eta_{p}^{2}=0.026 ; B F_{01}=2.29 ;$ Figure $\left.5 \mathrm{~B}\right)$. Next to these anticipatory effects, cluster-based permutation

531 tests identified significant below chance decoding during visual search using the distractor trained

532 weights ( $p<0.05$, cluster-corrected; dashed green horizontal lines). Together, these findings suggest 
bioRxiv preprint doi: https://doi.org/10.1101/2020.07.02.184945; this version posted December 9, 2020. The copyright holder for this preprint (which was not certified by peer review) is the author/funder, who has granted bioRxiv a license to display the preprint in perpetuity. It is made available under aCC-BY-NC-ND 4.0 International license.

533 that spatial frequency was differently represented in the pattern of scalp EEG activity as a function

534 of whether it was a feature of the target or of the distractor.

\section{5}

A
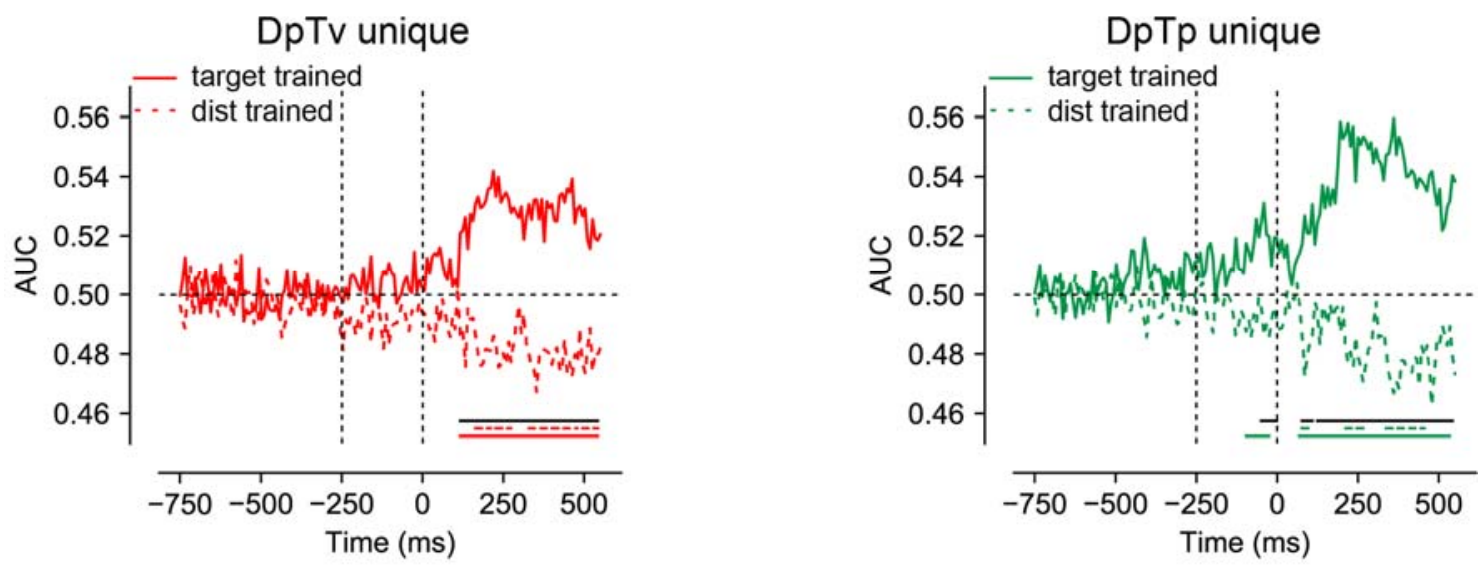

B

Pre-search display

Post-search display

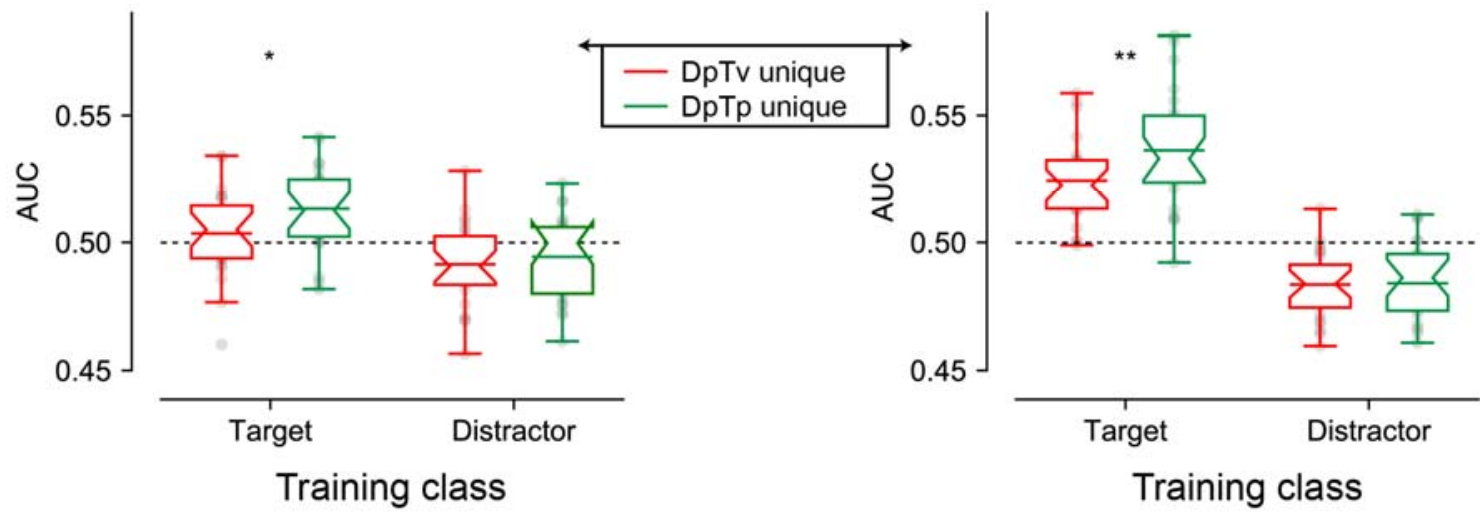

Figure 5 | Multivariate decoding of the target using either weights from a model trained on targets spatial

538 frequencies and distractor spatial frequencies. (A) AUC scores of DpTv unique (left) and DpTp unique (right) 539 decoding across time. Colored bars on the x-axis indicate clusters where conditions differ significantly from 540 chance after cluster correction ( $p<0.05$ ). Solid black lines indicate clusters with a significant difference between models trained on targets and distractors after cluster correction $(p<0.05)$. (B) Averaged auc scores during the pre_search display $(-250-0 \mathrm{~ms}$; left) and the post-search display $(0-550 \mathrm{~ms}$; right) visualized by notched boxplots with solid horizontal lines corresponding to the mean. Horizontal black dashed lines across plots indicates chance performance, whereas the vertical dashed black lines in the upper plots indicate the onset of placeholder (-250ms) and the search display (Oms).

\section{ERPs: The distractor Pd reduces as a function of spatial expectations}

549 Finally, we examined how distractor feature and locations expectations modulated stimulus 
551 (Eimer, 2014; Luck, 2012; Luck \& Hillyard, 1994) and suppression (Gaspelin \& Luck, 2018a; Hickey, Di

552 Lollo, \& McDonald, 2009), respectively. To isolate distractor- and target-specific components,

553 separate difference waveforms (contralateral - ipsilateral) were computed for lateralized distractors

554 below the horizontal midline with concurrent targets on the vertical midline, or vice versa, using

555 01/O2, PO3/PO4 and PO7/PO8 as electrodes of interest (see Methods section for details).

556 As visualized in Figure 6, we observed a clear distractor-evoked N2pc in all conditions, which

557 only seemed to be somewhat reduced in amplitude at the high probability distractor location in the

558 DpTp unique condition. Likewise, as shown in Figure 7, the target-evoked N2pc also appeared to be

559 virtually unaffected by feature and/or location expectations. Yet, notably, and in line with our

560 previous study (van Moorselaar \& Slagter, 2019), the distractor-evoked Pd was virtually eliminated

561 when distractors appeared at high probability distractor locations, signaling a reduced need for post-

562 distractor inhibition.

563 To statistically test these effects, voltages within the N2pc window (114 - 194ms; see

564 Methods section for details) were submitted to a Stimulus Class (target, distractor) by Condition

565 (DpTp identical, DpTp unique, DpTv unique) by Location (high distractor probability, low distractor

566 probability) by Hemifield (contralateral, ipsilateral) repeated measures ANOVA. This analysis

567 confirmed that the N2pc was reliably evoked across conditions (main effect Hemifield: $F(1,23)=$

$\left.56865.5, p<0.001, \eta_{p}^{2}=0.74\right)$ and also showed that as could be expected, the N2pc was more

569 pronounced in response to targets than distractors (interaction Stimulus Class by Hemifield: $F(1,23)$

$570=37.3, p<0.001, \eta_{p}^{2}=0.62$ ). Critically, in the DpTp unique condition, when participants had

571 foreknowledge about both the targets and distractors features, the distractor N2pc was reduced and

572 the target N2pc increased relative to the other conditions without unique target feature predictions

573 (interaction Stimulus Class by Condition by Hemifield: $F(2,46)=5.3, p=0.009, \eta_{p}^{2}=0.19$ ),

574 indicating that expectations at the target level made attentional selection more efficient. However,

575 there was no evidence whatsoever that location expectations modulated the amplitude of the N2pc,

576 neither for distractors or for targets (all $F^{\prime} s<1.1$, all $p^{\prime} s>0.33$, all $\eta_{p}^{2 \text { ‘s }}>0.048$ ). 
577

578

579

580

581

582

583

584

585

586

587

588

589

590

591

592

593

594

595

596

597

\section{DpTp identical}
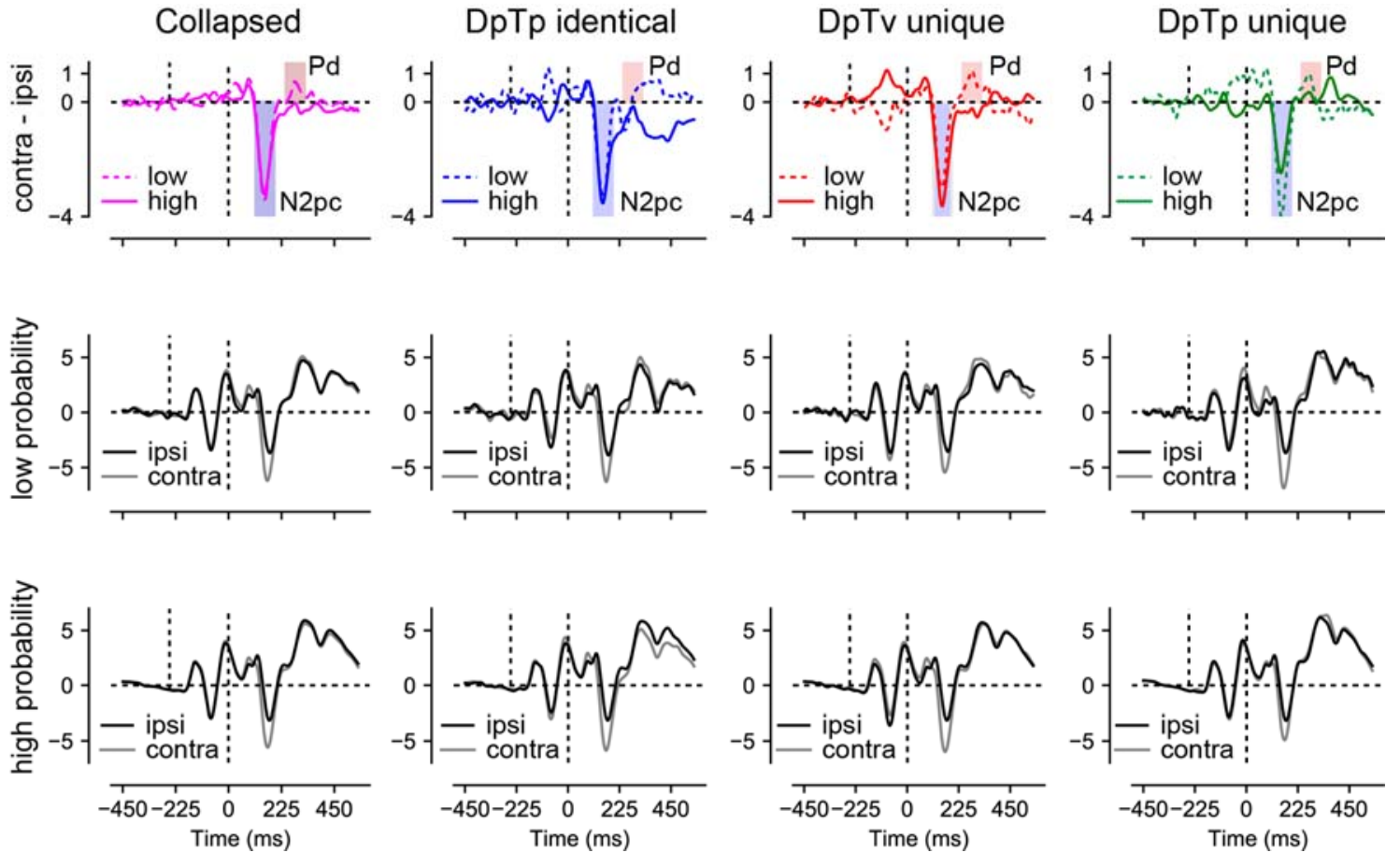

Figure 6| Distractor Pd reduces as a function of distractor location expectations. ERP results from search trials with lateral distractors collapsed across all conditions (column 1) and separately for each condition (columns 2 -4). Row 1 shows the difference between contra- and ipsilateral waveforms for the low and high probability distractor location. Only the distractor-evoked $\mathrm{Pd}$, not the $\mathrm{N} 2 \mathrm{pc}$, was reduced at high vs. low probability distractor locations (top left figure). Rows 2 and 3 show the contra- and ipsilateral waveforms for the low- and high probability distractor locations, respectively. Microvolts are plotted on the $y$-axes. Blue and red transparent rectangles in row 1 show the mean averaging windows for N2pc and Pd analyses.

The N2pc results suggest that distractors continued to capture attention to the same extent at high as at low probability distractor locations, albeit generally not as strongly as targets. Yet, at high probability locations there appeared to be a reduced need to actively counteract this shift of attention as evidenced by a reduction in the amplitude of the distractor-evoked $\mathrm{Pd}$. To statistically test this, average voltage values within the Pd window (243 - 323ms; see Methods section for details) elicited by distractors were submitted to a Condition (DpTp identical, DpTp unique, DpTv unique) by Location (high distractor probability, low distractor probability) by Hemifield (contralateral, ipsilateral) repeated measures ANOVA. Critically, this analysis yielded a Location by Hemifield interaction $\left(F(1,23)=5.1, p=0.033, \eta_{p}^{2}=0.18\right)$, reflecting a reduction in $\mathrm{Pd}$ amplitude at high vs. low probability distractor locations across conditions. Together these results replicate our previous findings that in a scenario where location expectations do not influence the initial selection 
598 of distracting information, they can nevertheless reduce the subsequent need to actively suppress

599 the expected distracting information (van Moorselaar \& Slagter, 2019). The main effect of Hemifield

600 and all other interaction effects with Location were non-significant (all $F^{\prime} s<1.9$, all $p$ 's $>0.16$, all $\eta_{p}^{2 \text { 's }}$

$601>0.077)$.
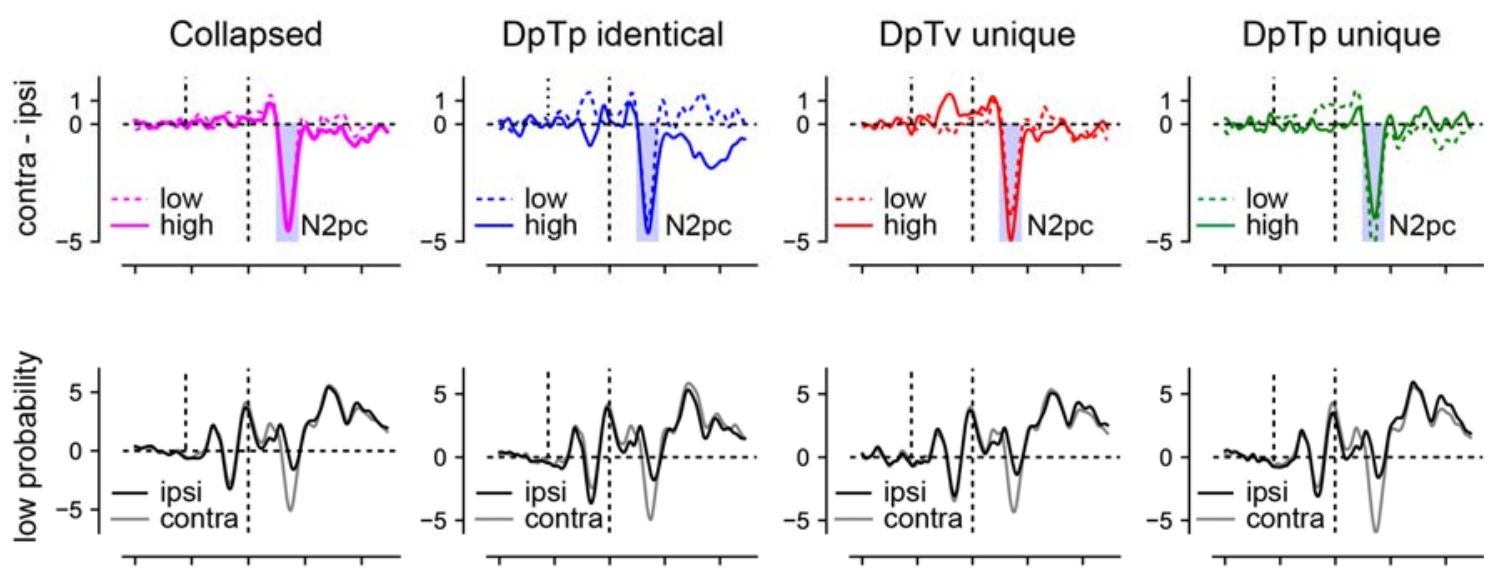

602
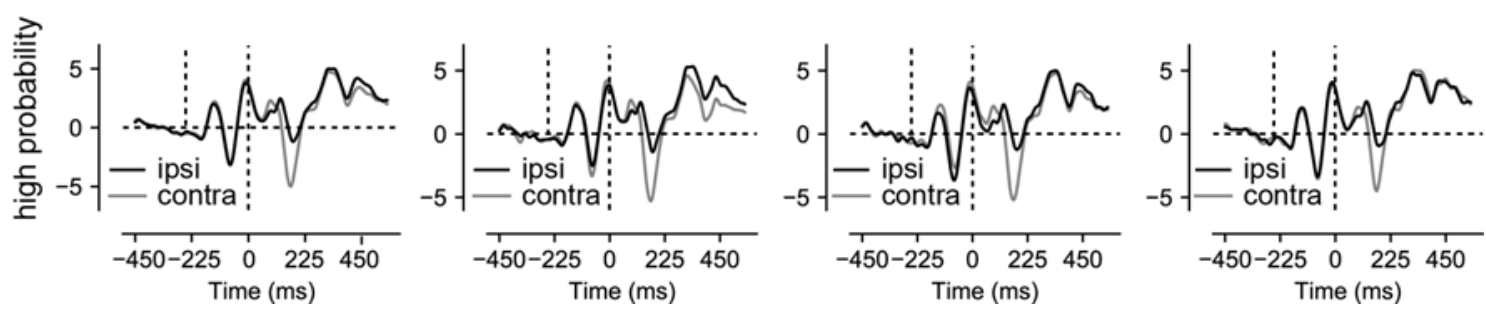

603

604

Figure 7 | No effect of distractor or target expectations on the target-evoked N2pc. This figure displays ERP results from search trials with lateral targets collapsed across all conditions (column 1 ) and separately for each condition (columns $2-4$ ) and the low- and high probability distractor locations (rows 2 and 3 , respectively).

606 Row 1 shows the difference between contra- and ipsilateral waveforms for low and high probability location.

607 Microvolts are plotted on the $y$-axes. Blue transparent rectangles in row 1 show the mean averaging windows

608 for N2pc analyses.

609

\section{Discussion}

611 The aim of the present study was to gain more insight into the neural mechanisms underlying

612 learning to ignore distracting information on the basis of spatial and/or non-spatial regularities.

613 While there is evidence that expectations based on regularities in the environment induce pre-

614 stimulus sensory templates (Kok, Mostert, \& De Lange, 2017; van Moorselaar \& Slagter, 2019), it

615 remains unclear whether the generation of such sensory templates is dependent on the task

616 relevance of the sensory information, and hence is also evident when distractor rather than target 
617 information can be predicted in advance. Here we manipulated the predictability of the distractor at

618 both the spatial and feature level, to address this outstanding question. At the behavioral level,

619 responses were faster when the distractor occurred at the high probability distractor location, but

620 slower when the target occurred at this location, relative to any other location, indicative of generic

621 expectation-dependent spatial suppression. Moreover, when distractors were also predictable at

622 the feature level, location-based distractor suppression became less pronounced, suggesting that

623 the locus of inhibition in the processing hierarchy is flexible and dependent on whether expectations

624 are location- and/or feature-specific. Yet, at the neural level, even when distractors were not

625 predictable at the feature level, we observed no evidence for preparatory spatial inhibition as a

626 function of distractor location learning, as indicated by the absence of a modulation of pre-stimulus

627 lateralized alpha-band activity. In contrast, when distractor features (their spatial frequency) were

628 predictable, they could already be decoded from the pattern of scalp EEG activity before search

629 display onset, potentially indicative of a distractor feature template. Strikingly, spatial frequency was

630 differently represented in the pattern of pre-stimulus scalp EEG activity as a function of whether it

631 was a predictable feature of the target or of the distractor, suggesting distinct target and distractor

632 templates. Spatial distractor expectations did reduce post-distractor inhibition, as indicated by a

633 strongly reduced distractor-evoked Pd. Together these findings demonstrate that location and

634 feature-based expectations in part rely on different neural mechanisms, but also interact, in

635 inhibiting distracting information.

636 At the spatial level, there is some evidence that pre-stimulus alpha-band activity contributes

637 to expectation-dependent suppression (Wang et al., 2019), but based on other work (Noonan et al.,

638 2016; van Moorselaar \& Slagter, 2019), we reasoned that this may be specific to conditions that

639 enforce learning at the spatial level. Counter to this idea, however, here lateralized alpha-band

640 activity was insensitive to distractor location learning, even when the target and distractor shared

641 the same spatial frequency and learning could only be location based. The absence of pre-stimulus

642 alpha-band modulations in any condition despite clear behavioral evidence for generic space-based 
643 suppression adds to a growing body of literature challenging the widely embraced view that

644 anticipatory distractor suppression is implemented via a spatially-specific increase in alpha-band

645 activity (van Moorselaar \& Slagter, 2020). Previously observed modulations of pre-stimulus alpha-

646 band activity may instead reflect attending away rather than suppression of distracting information,

647 as also argued previously (Foster \& Awh, 2018). Indeed, in our recent study using a similar visual

648 search task, but in which the target or the distractor was repeatedly presented at the same location,

649 across four consecutive trials, the topographic distribution of preparatory alpha power was sensitive

650 to the expected target location, but not to the expected distractor location (van Moorselaar \&

651 Slagter, 2019)(see also)(Noonan et al., 2016). Together, these findings suggest that target facilitation

652 and distractor suppression rely on distinct neural mechanisms, at least when space-based. Albeit

653 speculative, distractor location learning may be implemented via an activity silent mechanism or

654 synaptic efficiency (Stokes, 2015). An intuitively appealing idea, one that can also be easily

655 reconciled with proposals that (spatial) expectations exert their influence only after initial processing

656 of sensory input (Alilović, Timmermans, Reteig, Van Gaal, \& Slagter, 2019; Rao, DeAngelis, \& Snyder,

657 2012), is that distractor learning changes plasticity in priority maps of space (Ferrante et al., 2018).

658 Alternatively, it is possible that the spatial bias was evident in the neural signal, but our analysis,

659 which focused on lateralized alpha power, was simply not sensitive enough to detect such a bias.

660 Note, however, that in our previous work we also found no evidence for an anticipatory bias using

661 either a multivariate decoding or a forward encoding approach, although that study provided

662 considerably less time for distractor location predictions to develop (van Moorselaar \& Slagter,

663 2019). Nonetheless, in line with a large body of work relating changes in lateralized pre-stimulus

664 alpha-band activity to spatial attention (Foxe \& Snyder, 2011; Jensen \& Mazaheri, 2010), in that

665 study, the expected target location was represented in the pattern of pre-stimulus alpha-band

666 activity. Future work in humans and animals is thus necessary to determine whether changes in

667 synaptic plasticity indeed underlie distractor location learning, or alternatively whether spatial 
668 tuning can be observed in the absence of lateralized alpha modulations with more sensitive 669 methods, such as intracranial EEG.

670 A striking aspect of the present findings was that we did find evidence for distractor-specific

671 feature templates based on the pattern of pre-stimulus brain activity when the spatial frequency of

672 the distractor was predictable. The existence of so-called templates for rejection is a long-standing

673 topic of debate in the domain of working memory research (Arita et al., 2012; Beck \& Hollingworth,

674 2015). While behaviorally, there is some evidence in support of an advance distractor-specific

675 inhibitory template (e.g.,)(Carlisle \& Nitka, 2018; Woodman \& Luck, 2007), previous fMRI studies did

676 not provide support for the notion of a distinct distractor template (de Vries et al., 2019; Reeder et

677 al., 2018). Counter to these previous studies, which cued the distractor feature on a trial-by-trial

678 basis, hence promoting the use of visual working memory, here the distractor 'template' could be

679 formed on the basis of predictions derived from regularities in the search context. That we could

680 decode the predictable distractor feature under these conditions thus suggests that a distinct

681 distractor template may only arise as a function of learning from repeated encounters with the to-

682 be-ignored feature. The development of this template may rely on implicit learning mechanisms that

683 bypass working memory altogether (van Moorselaar \& Slagter, 2020) and hence should not be taken

684 as evidence for the existence of templates for rejection in working memory. Nevertheless, they do

685 convincingly show that distractor feature expectations, like target feature expectations, induce pre-

686 stimulus sensory templates.

687 Note, however, that decoding is uninformative about the underlying neural representation

688 (Naselaris \& Kay, 2015; van Moorselaar \& Slagter, 2020). The here observed anticipatory distractor

689 decoding could be driven by selective changes in the activity of neural populations representing the

690 predicted distractor feature, selective changes in the activity of neural populations representing

691 non-distractor or target (i.e., the other two spatial frequencies) features, or both. Indeed, it is well

692 known that the visual system can strategically boost features shifted away from the distractor

693 and/or target to increase discriminability between the target and the likely distractors (Geng \& 
694 Witkowski, 2019; Navalpakkam \& Itti, 2007). To determine whether the neural pattern underlying

695 distractor decoding was distinct from the pattern driving target decoding, we also examined

696 whether a model trained on distractor spatial frequencies generalized to target spatial frequencies.

697 Strikingly, we found that while we could decode the anticipated spatial frequency of the target

698 above chance in target-only trials prior to search display onset when the model was trained on

699 target spatial frequencies, we could not decode the same anticipated spatial frequency of the target

700 when the model was trained on distractor spatial frequencies. What's more, after search display

701 onset there was reliable below-chance decoding, which arguably arose because after search display

702 onset, attention was shifted to the target spatial frequency during training on the distractor spatial

703 frequency, resulting in negative decoding when the classifier was tested on the target-only trials and

704 the target now possessed the distractor spatial frequency. If distractor and target templates were

705 opposite of one another, however, because observers strategically shifted their attention away from

706 the anticipated distractor spatial frequency towards the remaining potential target spatial

707 frequencies, one would have expected to also observe reliable below chance decoding in the

708 anticipatory period. By contrast, pre-stimulus target decoding performance of the distractor-trained

709 model did not differ between the conditions in which the target spatial frequency could be predicted

710 in advance vs. could not be predicted in advance, nor was there below chance decoding. Although

711 these are exploratory null findings and therefore should be interpreted cautiously, they nevertheless

712 argue against the idea that attention was strategically shifted away towards one (or both) of the

713 remaining (and possible target) spatial frequencies, but rather support the notion of a distinct

714 distractor template. Note also that it is unlikely that the observed anticipatory distractor feature

715 decoding simply reflects inter-trial priming as in this scenario, one would not expect a difference in

716 the neural activity patterns driving distractor decoding versus target decoding, as was observed

717 here. Together, these decoding results indicate that target and distractor feature expectations are

718 implemented in distinct neural codes. Future M/EEG studies that apply forward encoding modeling 
719 are necessary to more precisely examine the nature of the changes in feature tuning that underlie

720 feature-based distractor suppression.

721 At the behavioral level, distractor interference was greatly reduced when distractors

722 occurred at the high probability distractor location. Yet, this benefit was not associated with active

723 anticipatory spatial suppression as implemented by alpha-band activity, as discussed above, nor did

724 we observe enhanced post-distractor suppression as indicated by the amplitude of the Pd ERP

725 component (Feldmann-Wüstefeld \& Vogel, 2019; Hickey et al., 2009). That is, replicating our

726 previous study (van Moorselaar \& Slagter, 2019), across conditions the distractor-evoked Pd was

727 strongly reduced when distractors appeared at their expected location, as if the brain had learned

728 that stimuli at those locations could be safely ignored, even though they still evoked a reliable N2pc.

729 While a reduction of the Pd appears consistent with a dampened response to expected sensory

730 input and thus less need for suppression, many studies have actually shown that expected

731 distractors, either at the spatial (Wang et al., 2019) or the feature level (Burra \& Kerzel, 2013;

732 Jannati et al., 2013), elicit a Pd instead of an N2pc. Following the idea that the Pd signals a

733 suppressive mechanism, which is stronger in contexts that require strong suppression (Hickey et al.,

734 2009), these findings are typically interpreted to reflect the suppression of salient distractors before

735 they capture attention (Gaspelin \& Luck, 2018b). To date, it is unclear why in certain contexts the Pd

736 increases, whereas in others, as in the current study, its amplitudes decreases as a function of

737 expectations (Heuer \& Schubö, 2019). Differences in distractor salience or in the presence of non-

738 singleton distractors may explain this discrepancy in findings. A better understanding of the factors

739 that modulate distractor learning and suppression is critical for advancing our understanding of

740 learned distractor suppression and should thus be the focus of future work.

741 Our ERP findings also corroborate the notion that the N2pc and the Pd reflect different

742 attentional mechanisms, as these components were differentially sensitive to feature and spatial

743 expectations. Although it was initially thought that the N2pc reflects a covert shift of attention to a

744 peripheral stimulus (Eimer, 1996; Woodman \& Luck, 1999), current evidence suggests that the N2pc 
745 indexes object individuation (Mazza \& Caramazza, 2015), the formation of an object representation

746 that is segregated from the background and other items in the display (Kahneman, Treisman, \&

747 Gibbs, 1992). This idea dovetails with our finding that N2pc amplitude was modulated by

748 expectations at the feature level, but not by spatial expectations. The specific perceptual process

749 reflected by the $\mathrm{N} 2 \mathrm{pc}$, however, continues to be the topic of active debate. In one perspective,

750 which is easily reconciled with the idea of object individuation, the N2pc reflects attentional

751 engagement, which according to Zivony, Allon, Luria, and Lamy (2018) reflects the deployment of

752 higher level processes that enable identification and binding of stimulus features, and, if necessary,

753 consolidation into working memory. Consistent with this perspective, here we show that when the

754 spatial frequency of both the target and the distractor were predictable, and distractors and targets

755 could thus easily be dissociated, the distractor-evoked N2pc was smaller in amplitude, whereas the

756 target-evoked N2pc increased in amplitude. By contrast, as outlined above, the Pd was only sensitive

757 to spatial expectations in that its amplitude was greatly reduced to distractors at the high probability

758 distractor location. This demonstrates that the $\mathrm{Pd}$, which is often used to index stimulus-driven

759 distractor inhibition (Gaspelin \& Luck, 2018b; Sawaki, Geng, \& Luck, 2012), is specifically sensitive to

760 learned expectations at the spatial level.

761 Although there is growing evidence that regularities at both the spatial and the feature level

762 shape attentional orienting, it is unclear to what extent, if at all (Wang \& Theeuwes, 2018b), feature-

763 based expectations can shape attentional selection above and beyond location-based suppression

764 (Stilwell et al., 2019). At the behavioral level, we found that distractor suppression at the high

765 probability distractor location, but not target suppression at the high probability distractor location,

766 was affected by stimulus feature regularities. This may suggest that while spatial distractor

767 expectations may induce feature-blind "proactive" suppression (which uniformly suppresses both

768 distractor and target processing at the expected distractor location), subsequent inhibition of stimuli

769 at the likely distractor location is dependent on expectations about what a distractor looks like at

770 the feature level. This makes sense from an ecological perspective: one would not want to simply 
771 inhibit anything occurring at a particular location in space. While the interaction between spatial and

772 feature expectations in the distractor tuned behavioral analyses was significant in both Experiments

7731 and 2, after priming effects were removed, it only remained significant in Experiment 2 . This may

774 suggest that the interaction also to some extent reflects an interaction between priming and

775 distractor feature expectations, but could also reflect the fact that Experiment 2 allowed for the

776 development of more robust feature and location expectations. That is, counter to Experiment 2, in

777 Experiment 1, conditions not only switched every block (and consequently also the predictable

778 distractor and/or target features), but participants also only completed 6 blocks per condition. In

779 Experiment 2, distractor location and feature learning could develop over a much longer time scale

780 (18 blocks), and participants completed 36 blocks per condition. Consequently, Experiment 1 may

781 have been less sensitive to detecting the interaction between feature and spatial expectations above

782 and beyond intertrial priming. The fact that the interaction remains reliable in Experiment 2 ,

783 however, suggests that these different types of expectations can interact above and beyond

784 intertrial biases.

785 It may seem surprising at first that none of our EEG measures showed an interaction

786 between spatial and feature expectations, while in the corresponding behavioral data, we found that

787 distractor interference was most reduced when both the location and the spatial frequency of the

788 distractor were predictable in advance. Yet, this may be explained by the fact that these EEG

789 measures are specifically tuned to capturing modulations in either spatial processing (i.e., spatially

790 lateralized measures (alpha asymmetry and Pd)) or feature representation (spatial frequency

791 decoding). As such, they may be less sensitive to revealing neural mechanisms that integrate spatial

792 and feature distractor foreknowledge. Indeed, our EEG data did show robust main effects of spatial

793 and feature expectations.

794 To summarize, our findings add to a rapidly growing body of work showing that the ability to

795 inhibit distracting information critically relies on expectations grounded in statistical regularities in

796 the environment. They extend this work by showing that feature-based distractor expectations, but 
797 not location-based distractor expectations, modulate pre-stimulus representations, and in a distinct

798 manner from expectations about upcoming target features, indicative of a distractor template.

799 These findings have important implications for cognitive (neuroscience) theories of selective

800 attention and predictive processing.

801

802

803

804

805

806

807

\section{Materials and methods}

808

809

810

811

812 A planned number of 18 participants (average $(M)$ age $=23$ years, range $19-27$; seven men)

813 participated in Experiment 1, and, after replacement of three participants (because preprocessing

814 resulted in exclusion of too many trials; details see below), a planned number of 24 participants $(M=$

81522 years old, age range $=19-26 ; 5$ men) participated in Experiment 2, in exchange for course credit

816 or monetary compensation (10 euros per hour). Participants reported normal or corrected-to-

817 normal vision and provided written informed consent prior to participation. The ethical committee

818 of the Department of Psychology of the University of Amsterdam approved the study (2018-BC-

819 9051), which was conformed to the Declaration of Helsinki.

\section{Task, stimuli and procedure}

\section{Experiment 1}

823 The paradigm was adapted from our initial study (van Moorselaar \& Slagter, 2019). As can be seen in

824 Figure 1, trials started with a fixation display (500-ms) that only contained a central black dot with a 825 white rim (radius $0.1^{\circ}$ ). A placeholder display was subsequently presented for $250-\mathrm{ms}$, after which a 
826 search display was shown for 200-ms or until response. The next trial started after response

827 collection, or in case of no response, 1000-ms after search display onset.

828 The placeholder display contained six black rimmed circles (radius $1.5^{\circ}$ ), all placed on an

829 imaginary circle (radius $4.6^{\circ}$ ) centered on fixation. At search display onset two Gabor patches

830 (contrast $=1$ ) were presented within the centers of two selected placeholders. One Gabor, the

831 target, was tilted left or right $\left(45^{\circ}\right.$ and $\left.135^{\circ}\right)$, while the other Gabor, the distractor, was vertically or

832 horizontally oriented. Although the target and the distractor could appear at all of six stimulus

833 positions, the distractor appeared on one particular location more frequently (70\%; location

834 counterbalanced across participants) than on any of the other locations. In each trial, the target

835 location was selected at random, once the distractor location was determined.

836 Crucially, the target and the distractor could either have a high $(s f=0.05)$ or a low $(s f=0.02)$

837 spatial frequency. In the DpTp identical condition, the distractor and the target had the same spatial

838 frequency (e.g., both high; counterbalanced across blocks). In the DpTp unique condition, the spatial

839 frequency of the target and the distractor differed, but they were also fixed within a block

840 (counterbalanced across blocks). In the DvTv unique condition, the target and distractor had

841 different spatial frequencies that varied from trial to trial (counterbalanced across trials). Thus, in

842 both the DpTp identical and DpTp unique condition, the target and distracter spatial frequency were

843 predictable in a given block, but not in the DvTv unique condition. Yet, only the DpTp unique

844 condition allowed for both distractor and target-specific predictions at the feature level.

845 Participants first completed 50 practice trials in which the target and the distractor had the 846 same spatial frequency, and then 18 experimental blocks (6 blocks for each condition) of 50 trials 847 each, with condition order (DpTp identical, DpTp unique, DvTv unique, ...., etc) counterbalanced over

848 participants. They were instructed to respond as fast as possible, while trying to keep the number of 849 errors to a minimum. At the end of each block, participants received feedback about response time 850 and accuracy and were encouraged to take a break. At start of each block, participants were 851 informed about the upcoming condition. 


\section{Experiment 2}

854 The task in Experiment 2 was similar to Experiment 1, but we included important changes (see

855 Figure 1). In the DpTp identical condition and in the DpTp unique condition, the spatial frequencies

856 of the target and distractor were again fixed and hence predictable within a block of trials, with the

857 main difference between conditions that in the former condition the target and distractor shared

858 the same spatial frequency, whereas in the latter their spatial frequencies differed and could guide

859 attentional selection. In the DpTv unique condition, the distractor and target also had unique spatial

860 frequencies, but only the spatial frequency of the distractor was fixed and hence predictable,

861 whereas the target spatial frequency was selected at random. Targets and distractors could have

862 one of three spatial frequencies (low $s f=0.02$; medium $s f=0.04$; high $s f=0.08$ ). To prevent a

863 systematic bias in the decoding analysis, in the DpTp and DpTv unique conditions, spatial

864 frequencies were selected such that across all blocks, a specific spatial frequency serving as a target

865 or a distractor was equally often coupled to the remaining spatial frequencies serving as a distractor

866 or a target, respectively. Also, in Experiment 2, to be able to focus on lateralized components in the

867 EEG signal, the high probability distractor location (i.e., $70 \%$ of distractor present trials) could only

868 be one of the lateralized locations below the horizontal midline. This location was fixed within a

869 condition and shifted to the other side of the display after all trials of a given condition were

870 completed, with the high probability location and condition order counterbalanced across

871 participants (e.g., 18 consecutive blocks DpTp identical, 18 consecutive blocks DpTv unique, 18

872 consecutive blocks DpTp unique). Finally, each block contained six randomly intermixed distractor

873 absent trials in which the target was presented in isolation at each location once.

874 Participants came to the lab twice. In each session, they completed 54 blocks of the task of

87556 trials each, wherein condition changed every 18 blocks. Simultaneously, we measured their brain

876 activity with EEG and their eye movements using eye tracking and EOG. In the first session,

877 participants first completed a series of 56 practice trials of the DpTp identical condition. Manual 
878 responses were collected via two purpose-built response buttons, which were positioned at the end

879 of the armrests of the participant's chair. In the search task, the length of the fixation display

880 preceding the placeholder display was randomly jittered between $650-\mathrm{ms}$ and $1000-\mathrm{ms}$.

881 In both experiments, a Windows 7 PC running OpenSesame v3 (Mathôt, Schreij, \&

882 Theeuwes, 2012) using PsychoPy (Peirce, 2009) functionality generated the stimuli on an ASUS

883 VG236 120-Hz monitor with a grey background, at $\sim 80 \mathrm{~cm}$ viewing distance. Participants sat in a

884 dimly lit room.

887 Analyses

888 Software

889 All preprocessing steps and most analyses were performed in a Python environment (Python

890 Software Foundation, https://www.python.org/). Preprocessing and subsequent analyses scripts are

891 publicly available and can be downloaded at https://github.com/dvanmoorselaar/DvM. These

892 custom written analysis scripts are largely based on MNE functionalities (Gramfort et al., 2014).

893 Repeated measures ANOVA and planned comparisons with paired t-tests were done using JASP

894 software (JASP-TEAM, 2018). For all non-significant results, we also report the Bayes factor to

895 quantify the evidence in favor of the null hypothesis $\left(\mathrm{BF}_{01}\right)$.

\section{Behavioral preprocessing}

898 As preregistered, behavioral analyses were limited to RT data of correct trials only. RTs were filtered

899 in a two-step trimming procedure: trials with RTs shorter than 200 ms were excluded, after which

900 data were trimmed on the basis of a cutoff value of 2.5 SD from the mean per participant. Exclusion

901 of incorrect responses (13.6\% in Experiment 1; 7.9\% in Experiment 2 ) and data trimming (2.0\% in

902 Experiment 1; 2.6\% in Experiment 2) resulted in an overall loss of $15.6 \%$ of the data in Experiment 1

903 and $10.5 \%$ of the data in Experiment 2. Analyses were either yoked to the distractor location, in 
904 which case trials with targets at the high probability distractor location were excluded, or to the

905 target location, in which case trials with distractors at the high probability location were excluded.

906 This control made sure reported effects were not inflated, but more specific to distractor and target

907 suppression, respectively, at the high probability distractor location.

908

909 EEG recording and preprocessing

910 EEG data were recorded at a sampling rate of $512 \mathrm{~Hz}$ using a 64-electrode cap with electrodes

911 placed according to the 10-10 system (Biosemi ActiveTwo system; biosemi.com) and from two

912 earlobes (used as offline reference). Vertical (VEOG) and horizontal EOG (HEOG) were recorded via

913 external electrodes placed $\sim 2 \mathrm{~cm}$ above and below the eye, and $\sim 1 \mathrm{~cm}$ lateral to the external canthi,

914 respectively. All preprocessing steps were performed on the EEG data of the two sessions

915 separately.

916 EEG data were re-referenced off-line to the average of the left and the right earlobe, and

917 subsequently high-pass filtered using a zero-phase 'firwin' filter at $0.1 \mathrm{~Hz}$ to remove slow drifts.

918 Epochs were then created from -750-ms to 550-ms surrounding search display onset, extended by

919 500-ms at the start and end of the epoch to control for filter artifacts during preprocessing and time-

920 frequency analysis. The resulting epochs were baseline normalized using the whole epoch as a

921 baseline to aid detection of noisy electrodes based on visual inspection. Prior to trial rejection

922 malfunctioning electrodes $(M=1.2$, range $=0-5)$ were temporarily removed based on visual

923 inspection. Epochs contaminated by EMG noise were identified using an adapted version of an

924 automatic trial-rejection procedure as implemented in the Fieldtrip toolbox (Oostenveld, Fries,

925 Maris, \& Schoffelen, 2011). To specifically identify epochs contaminated by muscle activity, we used

926 a 110-140 Hz band-pass filter, and allowed for variable z-score cut-offs per subjects based on within-

927 subject variance of z-scores (de Vries, van Driel, \& Olivers, 2017) resulting in the identification of on

928 average $10.2 \%$ of epochs (range $2.9 \%$ - 19.7\%) as potentially containing artifacts. Marked epochs

929 were visually inspected, resulting in an average rejection of $8.1 \%$ of epochs (range $1.4 \%-17.0 \%$ ). 
930 Next, ICA as implemented in the MNE 'extended-infomax' method was performed on non-epoched 1

$931 \mathrm{~Hz}$ high-pass filtered data to identify and remove eye-blink components from the epoched data.

932 Following ICA, malfunctioning electrodes identified earlier were interpolated using spherical splines

933 (Perrin, Pernier, Bertrand, \& Echallier, 1989), after which the EEG data of the separate sessions was

934 concatenated. Finally, we detected sudden jumps in the HEOG using a step algorithm, with a window

935 length of $200-\mathrm{ms}$, a step size of $10 \mathrm{~ms}$, and with a threshold of $20 \mu \mathrm{V}$. The detected jumps using

936 these settings were again visually inspected, which resulted in a rejection of $4.0 \%$ (range $0.4 \%$ -

$93711.4 \%)$ of the remaining epochs after artifact rejection ${ }^{2}$.

\section{Time-frequency analysis}

939 To isolate frequency-specific activity across time, we used Morlet wavelet convolution to

940 decompose EEG time series into their time-frequency representation for frequencies ranging from 1

941 to $40 \mathrm{~Hz}$ in 25 logarithmically spaced steps. To create complex Morlet wavelets, for each frequency a

942 sine wave ( $e^{i 2 \pi f t}$, where $i$ is the complex operator, $f$ is frequency, and $t$ is time) was multiplied by a

943 Gaussian $\left(e^{-t^{2} / 2 s^{2}}\right.$, where $s$ is the width of the Gaussian). To keep a good trade-off between

944 temporal and frequency precision the Gaussian width was set as $s=\delta /(2 \pi f)$, where $\delta$ represents

945 the number of wavelet cycles, in 25 logarithmically spaced steps between 3 and 12. Frequency-

946 domain convolution was then applied by multiplying the Fast Fourier Transform (FFT) of the EEG

947 data and Morlet wavelets. The resulting signal was converted back to the time domain using the

948 inverse FFT. Time-specific frequency power, which was downsampled by a factor of 4, was defined

949 as the squared magnitude of the complex signal resulting from the convolution.

950 To investigate whether the high probability distractor location resulted in changes in the

951 topographic distribution of alpha-power for each condition we calculated a lateralization index, in

952 which raw power in each hemisphere (i.e., contralateral and ipsilateral relative to the high

953 probability distractor location) is expressed relative to the total power at both sites. This number is

${ }^{2}$ Counter to the preregistration we did not use the EyeTribe data to exert control over which epochs to include in the analysis as in a large subset of subject's calibration required too much time, or did not work at all. We therefore relied on a two-step process where we visually inspected epochs selected by a step algorithm (for details see main text) 
954 positive when contralateral power is larger than ipsilateral power and negative when the inverse is

955 the case. This procedure yields a metric that shows interpretable dynamics over time and frequency

956 and has the benefit of not having to select a pre-stimulus interval (i.e., the interval that we are

957 interested in) for baseline normalization. Statistical analyses were limited to electrode pairs PO7/8

958 and $01 / 2$, which were selected on the basis of visual inspection of the topographic distribution of

959 averaged alpha-power $(8-12 \mathrm{~Hz})$ across the anticipatory time window $(-750-0 \mathrm{~ms})$.

960

961

962 Decoding analysis

963 To test whether feature-specific predictions modulated the neural representation of spatial

964 frequency, we applied multivariate pattern analysis (MVPA), using linear discriminant analysis

965 (Pedregosa et al., 2011) in combination with a 10-fold cross validation scheme, with all 64 channels

966 as features and target or distractor spatial frequency as classes. As we were specifically interested in

967 anticipatory effects, which we expected to be most pronounced, if at all present, immediately

968 preceding search display onset, preprocessed data was baseline corrected using a $-750 \mathrm{~ms}$ to $-550 \mathrm{~ms}$

969 window and subsequently down-sampled to $128 \mathrm{~Hz}$ to decrease the computational time of the

970 MVPA analysis. EEG data was randomly split into 10 equally sized subsets, while ensuring that each

971 class (i.e. low, medium, high spatial frequency) was selected equally often in each condition so that

972 training was not biased towards a specific class. Next, a leave-one-out procedure was used on the 10

973 folds, such that classifier was trained on 9 folds and tested on the remaining fold until each fold was

974 tested once. Classifier performance was then averaged over folds. As a measure of classifier

975 performance we used the Area Under the Curve (AUC), where a value of 0.5 is considered chance

976 classification, and which is considered a sensitive, nonparametric and criterion-free measure of

977 classification (Hand \& Till, 2001). This analysis was conducted for every time point, showing how

978 classifier performance changed over time.

979 


\section{$980 \quad$ ERP analysis}

981 For the ERP analysis we followed the same procedure as in our previous work (van Moorselaar \&

982 Slagter, 2019). To isolate distractor-specific ERP components, separate waveforms were computed

983 for ipsilateral and contralateral scalp regions for lateralized distractors presented below the

984 horizontal midline with concurrent targets on the vertical midline using 01/O2, PO3/PO4 and

$985 \mathrm{PO7/PO8}$ as electrodes of interest. Epochs were $30 \mathrm{~Hz}$ low-pass filtered and baseline corrected using

986 a 200-ms window preceding placeholder onset. The N2pc window was selected by taking the most

987 negative peak in the condition-averaged difference waveform (contralateral - ipsilateral) of both

988 targets and distractors in a $100 \mathrm{~ms}$ to $400 \mathrm{~ms}$ window post search display onset extended by $40 \mathrm{~ms}$ on

989 both sides of the peak. The same approach was used to determine the Pd window of interest, but

990 now searching the positive peak and only using distractor tuned waveforms.

991

\section{Statistics}

993 RTs, and averaged data across time windows of interest (in time-frequency, decoding and ERP) were

994 analyzed with repeated measures ANOVAs. Reported $p$-values are Greenhouse-Geiser corrected in

995 case of sphericity violations. In case of relevant significant effects, we followed up by planned

996 comparisons with paired t-tests, and in case of insignificant findings we report the Bayes factor to

997 evaluate the null hypothesis. Time windows with significant effects were identified using cluster-

998 based permutation testing across time and across time and frequency with cluster correction $(p=$

9990.05 and 1024 iterations) using MNE functionality (Gramfort et al., 2014)

1000

1001 References

1002 Alilović, J., Timmermans, B., Reteig, L. C., Van Gaal, S., \& Slagter, H. A. (2019). No evidence 1003 that predictions and attention modulate the first feedforward sweep of cortical 1004 information processing. Cerebral Cortex, 29(5), 2261-2278.

1005 Allenmark, F., Zhang, B., Liesefeld, H. R., Shi, Z., \& Müller, H. J. (2019). Probability cueing of 1006 singleton-distractor regions in visual search: the locus of spatial distractor 1007 suppression is determined by colour swapping. Visual Cognition, 1-19. 
1008

1009

1010

1011

1012

1013

1014

1015

1016

1017

1018

1019

1020

1021

1022

1023

1024

1025

1026

1027

1028

1029

1030

1031

1032

1033

1034

1035

1036

1037

1038

1039

1040

1041

1042

1043

1044

1045

1046

1047

1048

1049

1050

1051

1052

1053

1054

Arita, J. T., Carlisle, N. B., \& Woodman, G. F. (2012). Templates for rejection: configuring attention to ignore task-irrelevant features. Journal of Experimental Psychology: Human Perception and Performance, 38(3), 580.

Battistoni, E., Stein, T., \& Peelen, M. V. (2017). Preparatory attention in visual cortex. Annals of the New York Academy of Sciences, 1396(1), 92-107.

Beck, V. M., \& Hollingworth, A. (2015). Evidence for negative feature guidance in visual search is explained by spatial recoding. Journal of Experimental Psychology: Human Perception and Performance, 41(5), 1190.

Burra, N., \& Kerzel, D. (2013). Attentional capture during visual search is attenuated by target predictability: evidence from the $\mathrm{N} 2 \mathrm{pc}, \mathrm{Pd}$, and topographic segmentation. Psychophysiology, 50(5), 422-430.

Carlisle, N. B., \& Nitka, A. W. (2018). Location-based explanations do not account for active attentional suppression. Visual Cognition, 1-12.

Chelazzi, L., Marini, F., Pascucci, D., \& Turatto, M. (2019). Getting rid of visual distractors: The why, when, how and where. Current opinion in psychology.

Cunningham, C. A., \& Egeth, H. E. (2016). Taming the white bear: Initial costs and eventual benefits of distractor inhibition. Psychological Science, 27(4), 476-485.

de Vries, I. E., Savran, E., van Driel, J., \& Olivers, C. N. (2019). Oscillatory mechanisms of preparing for visual distraction. Journal of cognitive neuroscience, 1-22.

de Vries, I. E., van Driel, J., \& Olivers, C. N. (2017). Posterior alpha EEG dynamics dissociate current from future goals in working memory guided visual search. Journal of Neuroscience, 2945-2916.

Eimer, M. (1996). The N2pc component as an indicator of attentional selectivity. Electroencephalography and clinical neurophysiology, 99(3), 225-234.

Eimer, M. (2014). The neural basis of attentional control in visual search. Trends in cognitive sciences, $18(10)$, 526-535.

Failing, M., Feldmann-Wustefeld, T., Wang, B., Christian, O., \& Theeuwes, J. (2019). Statistical regularities induce spatial as well as feature-specific suppression. Journal of Experimental Psychology: Human Perception and Performance.

Failing, M., Wang, B., \& Theeuwes, J. (2019). Spatial suppression due to statistical regularities is driven by distractor suppression not by target activation. Attention, Perception, \& Psychophysics, 1-10.

Feldmann-Wüstefeld, T., \& Vogel, E. K. (2019). Neural evidence for the contribution of active suppression during working memory filtering. Cerebral Cortex, 29(2), 529-543.

Ferrante, O., Patacca, A., Di Caro, V., Della Libera, C., Santandrea, E., \& Chelazzi, L. (2018). Altering spatial priority maps via statistical learning of target selection and distractor filtering. Cortex, 102, 67-95.

Foster, J. J., \& Awh, E. (2018). The role of alpha oscillations in spatial attention: Limited evidence for a suppression account. Current opinion in psychology.

Foxe, J. J., \& Snyder, A. C. (2011). The role of alpha-band brain oscillations as a sensory suppression mechanism during selective attention. Frontiers in psychology, 2, 154. doi:ARTN 154

10.3389/fpsyg.2011.00154

Friston, K. (2009). The free-energy principle: a rough guide to the brain? Trends in cognitive sciences, 13(7), 293-301.

Gaspar, J. M., \& McDonald, J. J. (2014). Suppression of salient objects prevents distraction in visual search. Journal of Neuroscience, 34(16), 5658-5666. 
1055

1056

1057

1058

1059

1060

1061

1062

1063

1064

1065

1066

1067

1068

1069

1070

1071

1072

1073

1074

1075

1076

1077

1078

1079

1080

1081

1082

1083

1084

1085

1086

1087

1088

1089

1090

1091

1092

1093

1094

1095

1096

1097

1098

1099

1100

Gaspelin, N., \& Luck, S. J. (2018a). Combined Electrophysiological and Behavioral Evidence for the Suppression of Salient Distractors. Journal of cognitive neuroscience, 1-16.

Gaspelin, N., \& Luck, S. J. (2018b). The role of inhibition in avoiding distraction by salient stimuli. Trends in cognitive sciences, 22(1), 79-92.

Geng, J. J., \& Witkowski, P. (2019). Template-to-distractor distinctiveness regulates visual search efficiency. Current opinion in psychology.

Giesbrecht, B., Weissman, D. H., Woldorff, M. G., \& Mangun, G. R. (2006). Pre-target activity in visual cortex predicts behavioral performance on spatial and feature attention tasks. Brain research, 1080(1), 63-72.

Goschy, H., Bakos, S., Müller, H. J., \& Zehetleitner, M. (2014). Probability cueing of distractor locations: both intertrial facilitation and statistical learning mediate interference reduction. Frontiers in psychology, 5, 1195.

Gramfort, A., Luessi, M., Larson, E., Engemann, D. A., Strohmeier, D., Brodbeck, C., . . . Hämäläinen, M. S. (2014). MNE software for processing MEG and EEG data. Neuroimage, 86, 446-460.

Hand, D. J., \& Till, R. J. (2001). A simple generalisation of the area under the ROC curve for multiple class classification problems. Machine learning, 45(2), 171-186.

Heuer, A., \& Schubö, A. (2019). Cueing distraction: electrophysiological evidence for anticipatory active suppression of distractor location. Psychological research, 1-11.

Hickey, C., Di Lollo, V., \& McDonald, J. J. (2009). Electrophysiological indices of target and distractor processing in visual search. Journal of cognitive neuroscience, 21(4), 760775.

Hopfinger, J. B., Buonocore, M. H., \& Mangun, G. R. (2000). The neural mechanisms of topdown attentional control. Nature neuroscience, 3(3), 284-291.

Jannati, A., Gaspar, J. M., \& McDonald, J. J. (2013). Tracking target and distractor processing in fixed-feature visual search: evidence from human electrophysiology. Journal of Experimental Psychology: Human Perception and Performance, 39(6), 1713.

JASP-TEAM. (2018). JASP (Version 0.8.2) [Computer software].

Jensen, O., \& Mazaheri, A. (2010). Shaping functional architecture by oscillatory alpha activity: gating by inhibition. Frontiers in human neuroscience, 4, 186.

Kahneman, D., Treisman, A., \& Gibbs, B. J. (1992). The reviewing of object files: Objectspecific integration of information. Cognitive psychology, 24(2), 175-219.

Kenemans, J., Baas, J., Mangun, G. R., Lijffijt, M., \& Verbaten, M. (2000). On the processing of spatial frequencies as revealed by evoked-potential source modeling. Clinical neurophysiology, 111(6), 1113-1123.

Kenemans, J., Kok, A., \& Smulders, F. (1993). Event-related potentials to conjunctions of spatial frequency and orientation as a function of stimulus parameters and response requirements. Electroencephalography and Clinical Neurophysiology/Evoked Potentials Section, 88(1), 51-63.

Kok, P., Mostert, P., \& De Lange, F. P. (2017). Prior expectations induce prestimulus sensory templates. Proceedings of the National Academy of Sciences, 201705652.

Kok, P., Rahnev, D., Jehee, J. F., Lau, H. C., \& De Lange, F. P. (2012). Attention reverses the effect of prediction in silencing sensory signals. Cerebral Cortex, 22(9), 2197-2206.

Luck, S. J. (2012). Electrophysiological correlates of the focusing of attention within complex visual scenes: N2pc and related ERP components. The Oxford handbook of eventrelated potential components, 329-360. 
1101

1102

1103

1104

1105

1106

1107

1108

1109

1110

1111

1112

1113

1114

1115

1116

1117

1118

1119

1120

1121

1122

1123

1124

1125

1126

1127

1128

1129

1130

1131

1132

1133

1134

1135

1136

1137

1138

1139

1140

1141

1142

1143

1144

1145

1146

1147

Luck, S. J., \& Hillyard, S. A. (1994). Electrophysiological correlates of feature analysis during visual search. Psychophysiology, 31(3), 291-308.

Maljkovic, V., \& Nakayama, K. (1994). Priming of pop-out: I. Role of features. Memory \& cognition, 22(6), 657-672.

Mathôt, S., Schreij, D., \& Theeuwes, J. (2012). OpenSesame: An open-source, graphical experiment builder for the social sciences. Behavior research methods, 44(2), 314324.

Mazza, V., \& Caramazza, A. (2015). Multiple object individuation and subitizing in enumeration: a view from electrophysiology. Frontiers in human neuroscience, 9, 162.

Naselaris, T., \& Kay, K. N. (2015). Resolving ambiguities of MVPA using explicit models of representation. Trends in cognitive sciences, 19(10), 551-554.

Navalpakkam, V., \& Itti, L. (2007). Search goal tunes visual features optimally. Neuron, 53(4), 605-617.

Noonan, M. P., Adamian, N., Pike, A., Printzlau, F., Crittenden, B. M., \& Stokes, M. G. (2016). Distinct mechanisms for distractor suppression and target facilitation. Journal of Neuroscience, 36(6), 1797-1807.

Noonan, M. P., Crittenden, B. M., Jensen, O., \& Stokes, M. G. (2018). Selective inhibition of distracting input. Behavioural brain research, 355, 36-47.

Oostenveld, R., Fries, P., Maris, E., \& Schoffelen, J.-M. (2011). FieldTrip: open source software for advanced analysis of MEG, EEG, and invasive electrophysiological data. Computational intelligence and neuroscience, 2011, 1.

Park, S., Kim, M.-S., \& Chun, M. M. (2007). Concurrent working memory load can facilitate selective attention: evidence for specialized load. Journal of Experimental Psychology: Human Perception and Performance, 33(5), 1062.

Pedregosa, F., Varoquaux, G., Gramfort, A., Michel, V., Thirion, B., Grisel, O., . . Dubourg, V. (2011). Scikit-learn: Machine learning in Python. Journal of machine learning research, 12(Oct), 2825-2830.

Peirce, J. W. (2009). Generating stimuli for neuroscience using PsychoPy. Frontiers in neuroinformatics, 2, 10.

Perrin, F., Pernier, J., Bertrand, O., \& Echallier, J. (1989). Spherical splines for scalp potential and current density mapping. Electroencephalography and clinical neurophysiology, 72(2), 184-187.

Proverbio, A. M., Zani, A., \& Avella, C. (1996). Differential activation of multiple current sources of foveal VEPs as a function of spatial frequency. Brain Topography, 9(1), 5968.

Rao, V., DeAngelis, G. C., \& Snyder, L. H. (2012). Neural correlates of prior expectations of motion in the lateral intraparietal and middle temporal areas. Journal of Neuroscience, 32(29), 10063-10074.

Reder, L. M., Weber, K., Shang, J., \& Vanyukov, P. M. (2003). The adaptive character of the attentional system: statistical sensitivity in a target localization task. Journal of Experimental Psychology: Human Perception and Performance, 29(3), 631.

Reeder, R. R., Olivers, C. N., Hanke, M., \& Pollmann, S. (2018). No evidence for enhanced distractor template representation in early visual cortex. Cortex; a journal devoted to the study of the nervous system and behavior, 108, 279-282.

Sauter, M., Liesefeld, H. R., Zehetleitner, M., \& Müller, H. J. (2018). Region-based shielding of visual search from salient distractors: Target detection is impaired with same-but 
1148

1149

1150

1151

1152

1153

1154

1155

1156

1157

1158

1159

1160

1161

1162

1163

1164

1165

1166

1167

1168

1169

1170

1171

1172

1173

1174

1175

1176

1177

1178

1179

1180

1181

1182

1183

1184

1185

1186

1187

1188

1189

1190 not different-dimension distractors. Attention, Perception, \& Psychophysics, 80(3), 622-642.

Sawaki, R., Geng, J. J., \& Luck, S. J. (2012). A common neural mechanism for preventing and terminating the allocation of attention. Journal of Neuroscience, 32(31), 1072510736.

Sawaki, R., \& Luck, S. J. (2010). Capture versus suppression of attention by salient singletons: Electrophysiological evidence for an automatic attend-to-me signal. Attention, Perception, \& Psychophysics, 72(6), 1455-1470.

Stilwell, B. T., Bahle, B., \& Vecera, S. P. (2019). Feature-based statistical regularities of distractors modulate attentional capture. Journal of Experimental Psychology: Human Perception and Performance, 45(3), 419.

Stokes, M. G. (2015). 'Activity-silent'working memory in prefrontal cortex: a dynamic coding framework. Trends in cognitive sciences, 19(7), 394-405.

van Driel, J., Olivers, C. N., \& Fahrenfort, J. J. (2019). High-pass filtering artifacts in multivariate classification of neural time series data. bioRxiv, 530220.

van Moorselaar, D., \& Slagter, H. A. (2019). Learning What Is Irrelevant or Relevant: Expectations Facilitate Distractor Inhibition and Target Facilitation through Distinct Neural Mechanisms. Journal of Neuroscience, 39(35), 6953-6967. doi:https://doi.org/10.1523/JNEUROSCI.0593-19.2019

van Moorselaar, D., \& Slagter, H. A. (2020). Inhibition in selective attention. Annals of the New York Academy of Sciences.

Vatterott, D. B., \& Vecera, S. P. (2012). Experience-dependent attentional tuning of distractor rejection. Psychonomic Bulletin \& Review, 19(5), 871-878.

Wang, B., \& Theeuwes, J. (2018a). Statistical regularities modulate attentional capture. Journal of Experimental Psychology: Human Perception and Performance, 44(1), 13.

Wang, B., \& Theeuwes, J. (2018b). Statistical regularities modulate attentional capture independent of search strategy. Attention, Perception, \& Psychophysics, 80(7), 17631774.

Wang, B., van Driel, J., Ort, E., \& Theeuwes, J. (2019). Anticipatory distractor suppression elicited by statistical regularities in visual search. Journal of cognitive neuroscience, 1-14.

Woodman, G. F., \& Luck, S. J. (1999). Electrophysiological measurement of rapid shifts of attention during visual search. Nature, 400(6747), 867-869.

Woodman, G. F., \& Luck, S. J. (2007). Do the contents of visual working memory automatically influence attentional selection during visual search? Journal of Experimental Psychology: Human Perception and Performance, 33(2), 363.

Zhang, B., Allenmark, F., Liesefeld, H. R., Shi, Z., \& Muller, H. (2019). Probability cueing of singleton-distractor locations in visual search: priority-map-or dimension-based inhibition? Journal of Experimental Psychology: Human Perception and Performance.

Zivony, A., Allon, A. S., Luria, R., \& Lamy, D. (2018). Dissociating between the N2pc and attentional shifting: An attentional blink study. Neuropsychologia, 121, 153-163. 